\title{
The guanine nucleotide exchange factor Net1 facilitates the specification of dorsal cell fates in zebrafish embryos by promoting maternal $\beta$-catenin activation
}

\author{
Shi Wei ${ }^{1}$, Miaomiao Dai ${ }^{1}$, Zhaoting Liu ${ }^{1}$, Yuanqing $\mathrm{Ma}^{1}$, Hanqiao Shang ${ }^{1}$, Yu Cao ${ }^{1}$, Qiang Wang ${ }^{1,2}$ \\ ${ }^{I}$ State Key Laboratory of Membrane Biology, CAS Center for Excellence in Molecular Cell Science, Institute of Zoology, Chi- \\ nese Academy of Sciences, Beijing 100101, China; ${ }^{2}$ Savaid Medical School, University of Chinese Academy of Sciences, Beijing \\ 100049, China
}

Wnt/ $\beta$-catenin signaling is essential for the initiation of dorsal-ventral patterning during vertebrate embryogenesis. Maternal $\beta$-catenin accumulates in dorsal marginal nuclei during cleavage stages, but its critical target genes essential for dorsalization are silent until mid-blastula transition (MBT). Here, we find that zebrafish net1, a guanine nucleotide exchange factor, is specifically expressed in dorsal marginal blastomeres after MBT, and acts as a zygotic factor to promote the specification of dorsal cell fates. Loss- and gain-of-function experiments show that the GEF activity of Net1 is required for the activation of $\mathbf{W n t} / \beta$-catenin signaling in zebrafish embryos and mammalian cells. Net1 dissociates and activates PAK1 dimers, and PAK1 kinase activation causes phosphorylation of S675 of $\beta$-catenin after MBT, which ultimately leads to the transcription of downstream target genes. In summary, our results reveal that Net1-regulated $\beta$-catenin activation plays a crucial role in the dorsal axis formation during zebrafish development.

Keywords: Net1; dorsal axis formation; $\beta$-catenin phosphorylation; PAK1 dimerization; zebrafish Cell Research (2017) 27:202-225. doi:10.1038/cr.2016.141; published online 2 December 2016

\section{Introduction}

A conserved feature of all vertebrate embryos is the establishment of a basic body plan, which includes the specification of both the anterior-posterior and dorsal-ventral axes $[1,2]$. Previous studies have found that maternal $\mathrm{Wnt} / \beta$-catenin signaling is required for the initiation of dorsal-ventral patterning in the developing embryos [3-8]. $\beta$-catenin is the major effector of the canonical Wnt signaling pathway, and its stability and activity determine subsequent expression of downstream target genes. When Wnt signal is absent, $\beta$-catenin is degraded by a destruction complex consisting of Axin, adenomatous polyposis coli protein, casein kinase $1 \alpha(\mathrm{CK} 1 \alpha)$

Correspondence: Qiang Wang

Tel: +86-10-6480-7895; Fax: +86-10-6480-7895

E-mail: qiangwang@ioz.ac.cn

Received 21 May 2016; revised 18 September 2016; accepted 27 September 2016; published online 2 December 2016 and glycogen synthase kinase-3 $\beta$ (GSK-3 $\beta$ ), which phosphorylates $\beta$-catenin on $\mathrm{N}$-terminal serine and threonine residues for ubiquitin-mediated degradation [9-11]. The presence of the Wnt ligand inhibits the degradation complex and causes $\beta$-catenin stabilization and subsequent translocation to the nucleus, where it activates target gene transcription $[12,13]$. In anamniotes, following fertilization, a microtubule-dependent movement leads to the asymmetric localization of maternal Wnt ligands, which triggers the accumulation of $\beta$-catenin in the blastomeres of the prospective dorsal side during cleavage stages $[4,14]$. Although this asymmetrical $\beta$-catenin accumulation activates the transcription of a small number of genes before the mid-blastula transition (MBT) [15], critical target genes that inhibit the action of ventralizing factors, including bozozok (boz) and chordin (chd), are not expressed until after the MBT [1, 2, 16-19]. These observations suggest that the transcriptional activity of nuclear $\beta$-catenin may be limited before MBT. Further elucidation of the cellular modification and subsequent $\beta$-catenin activation is crucial in understanding these de- 
velopmental processes.

Phosphorylation of $\beta$-catenin not only causes its degradation, but has also been shown to induce other effects on its cellular function [20]. In addition to phosphorylation by GSK-3 $\beta$ and CK1 $\alpha$ at the $N$ terminus, $\beta$-catenin can also be phosphorylated at S552, Y654, S663 and S675, resulting in enhanced protein stability or transcriptional activity [20-24]. However, the developmental roles of these $\mathrm{C}$-terminal modifications of $\beta$-catenin in vertebrate dorsal axis formation have not yet been elucidated.

Accumulating evidence indicates that the Rho GTPase-mediated phosphorylation of $\beta$-catenin plays a vital role in regulating Wnt signaling. RhoA, Rac1 and Cdc42 belong to Rho family of small GTPases, and act as molecular switches that cycle between an inactive GDPbound state and an active GTP-bound state. The activation of Rho GTPases is tightly controlled by specific guanine nucleotide exchange factors (GEFs) that stimulate the exchange of GDP for GTP, which is important for many actin-dependent processes such as cell migration, adhesion and morphogenesis $[25,26]$. It has been shown that Rac1 activates JNK2 that in turn phosphorylates $\beta$-catenin at Ser191 and Ser605 and controls $\beta$-catenin nuclear translocation in the mouse bone marrow-derived ST2 cell line [27]. Rac1 also activates p21-activated kinase 1 (PAK1) to promote $\beta$-catenin S675 phosphorylation and activation in colon cancer cells [23]. A number of GEFs for the Rho family of small GTPases also play vital role in regulating $\mathrm{Wnt} / \beta$-catenin signal. In colorectal cancer cells, Rac1 exchange factor Tiam1 associates with Wnt-responsive promoters in response to Wnt ligand stimulation and activates nuclear Rac1 to enhance Wnt target gene transcription [28]. In colon cancer and HEK 293 cells, DOCK4 and $\beta_{1}$ Pix, two other Rac GEFs, can also promote canonical Wnt signaling by enhancing either the stability or transcriptional activity of $\beta$-catenin $[29,30]$. Furthermore, a recent study indicated that Rho GEF Pebble/ECT2 plays a conserved role in inhibiting Wnt activity downstream of $\beta$-catenin stabilization, and that loss and gain of its function in Drosophila embryos cause patterning defects [31]. Our previous study revealed that one such GEF, neuroepithelial cell transforming 1 (net 1 ), is highly expressed in the pre-dorsal organizer at the onset of gastrulation in the zebrafish embryo [32]. Given that net1 is spatially and temporally expressed in a region of the zebrafish embryo in which Wnt signaling is known to play a prominent role during development, it seems plausible that Net1 is likely to be involved in the $\mathrm{Wnt} / \beta$-catenin pathway.

Net1 is a RhoA-specific GEF originally isolated from neuroepithelioma cells as a novel oncogene [33]. Net1 protein contains a catalytic Dbl homology (DH) do- main and an adjacent pleckstrin homology (PH) domain flanked by $\mathrm{N}$ - and C-terminal extensions [34]. The DH and $\mathrm{PH}$ domains are necessary for binding to the GTPase and stimulating nucleotide exchange activity [34]. Because Net1 possesses two nuclear localization signal (NLS) sequences in its N-terminus, wild-type Net1 mainly resides in the nucleus [35], but Net1 can enter the cytoplasm, and only cytoplasmic Net1 activates RhoA and induces stress fiber formation [36]. Mutation in NLS or deletion of the $\mathrm{N}$-terminus resulted in a partial redistribution of Net1 to the cytosol $[35,36]$. Therefore, nuclear localization of Net1 provides a potential mechanism for sequestering GEF away from RhoA [35]. However, nuclear Net1 also exists in an active form and has been reported to increase nuclear RhoB activity upon treatment with DNA damaging agents [37], but its physiological roles are not well defined.

Net1 and RhoA have been shown to play important roles in various aspects of vertebrate embryonic development and organogenesis. In Xenopus, Net1 associates with Dishevelled and activates RhoA to regulate gastrulation [38]. In chick gastrulation-stage embryos, reducing Net1 or RhoA expression in epiblast cells prior to the epithelial-to-mesenchymal transition (EMT) leads to ingression and migration defects [39]. In mouse embryos, net 1-deficient mammary glands exhibit decreased RhoA-mediated phosphorylation of the regulatory subunits of myosin light chain and myosin light-chain phosphatase, which results in reduced and disorganized ductal branching [40]. Furthermore, in zebrafish, RhoA has been shown to control convergence and extension (CE) during gastrulation [41]. However, the developmental role of Net1 has never been assessed in zebrafish embryonic development. While our previous study showed that net 1 is expressed in the pre-dorsal organizer of the zebrafish embryo [32], the mechanism by which Net1 elicits downstream effects remains to be elucidated.

Here, we demonstrate that Net1 regulates the phosphorylation of $\beta$-catenin at S675, which is essential for the induction of downstream $\beta$-catenin transcriptional activity that specifies dorsal cell fates. Net1 acts upstream of PAK1 to promote $\beta$-catenin phosphorylation during early embryonic development. Specifically, we show that Net1, via an unidentified GTPase, dissociates and activates PAK1 dimers, which in turn phosphorylate $\beta$-catenin at the S675 site. Therefore, we provide direct evidence of a regulatory cascade consisting of Net1GTPase-PAK1 that controls canonical Wnt signaling, and demonstrate that the C-terminal phosphorylation of $\beta$-catenin is a critical requirement for dorsal development of zebrafish embryos. 


\section{Results}

Zebrafish net1 is essential for organizer formation and dorsal fate specification

Mammalian Net1 was found to be a RhoA-specific GEF that is upregulated in many carcinomas to enhance cell migration and invasion [42-46], but its function during embryonic development has not been fully described. Zebrafish net1, which encodes a protein sharing a similar domain architecture and a high sequence identity with its human and mouse orthologs $(57.17 \%$ and $57.66 \%$, respectively), was first identified as a dorsally expressed gene at the onset of gastrulation [32]. Wholemount RNA in situ hybridization revealed that zebrafish net 1 transcript was absent during maternal stages and was first detected in a dorsoventral gradient in the blastoderm margin with the highest level in the dorsal side at $30 \%$ epiboly stage (Figure 1A). At shield stage, net1 expression became more restricted to the dorsal organizer, and retained a lower level in the lateral margin (Figure 1A). Consistent with its organizer expression, net 1 was most notably expressed in the axial mesoderm at mid-gastrulation stage (75\% epiboly; Supplementary information, Figure S1A). Interestingly, as segmentation proceeds during somitogenesis, the expression domain of net 1 moves to the presomitic mesoderm (Supplementary information, Figure S1A). In addition, western blot analyses revealed that Net1 protein was absent in maternal stages, but started to be expressed before gastrulation (Figure 1B). By using a more sensitive detection system, semi-quantitative RT-PCR, we found that zebrafish embryos began to express net 1 transcript around MBT (1K-cell stage; Figure 1C). These results indicate that net 1 is an early zygotic gene which may have potential roles in organizer formation and dorsal fate specification.

As net 1 is one of the previously identified Nodal/ Smad2 target genes at the onset of gastrulation [32], we next explored whether the expression of net 1 at the dorsal blastoderm margin is regulated by Nodal signaling. As shown in Supplementary information, Figure S1B, the expression of net 1 was abolished in Nodal-deficient MZoep mutants at the $30 \%$-epiboly and shield stages. In contrast, the dorsal expression domain of net 1 was dramatically expanded in the Nodal ligand squint (sqt) mRNA injected embryos (Supplementary information, Figure S1C). In addition, net 1 expression was also obviously decreased in MZoep mutants revealed by semi-quantitative RT-PCR at sphere stage, the essential period for dorsal cell fate specification (Supplementary information, Figure S1D). These results suggest that Nodal signal activates the expression of net 1 in the pre-dorsal organizer.
In order to investigate the developmental function of net1, we first used morpholino-based gene knockdown technology to disrupt net 1 expression in wild-type zebrafish embryos. Two splice-blocking morpholinos (MO1 and MO2) targeting different exon-intron boundaries of net 1 pre-mRNA were designed and synthesized. Compared with mismatched control morpholino (cMO) injection, the injection of $4 \mathrm{ng}$ of either MO1 or MO2 efficiently blocked the expression of endogenous net 1 mRNA and protein, and only the interfered mRNA products were detected in the morphants (Supplementary information, Figure S2A and S2B). These results indicate the specificity and efficiency of net 1 MOs. Embryos injected with different amounts of MO1 exhibited distinct dorsal defects in a dose-dependent manner during body plan formation, including a smaller organizer at shield stage, a variably reduced head size and shortened body axis at $24 \mathrm{hpf}$ (hours post fertilization; Supplementary information, Figure S2C). Likewise, injection of MO2 also led to similar defects in dorsal axis formation (Supplementary information, Figure S2D).

In addition to the morphological defects in net 1 morphants, expression of dorsal markers, such as boz, chd and goosecoid (gsc) was significantly decreased at sphere and shield stages (Figure 1D-1E' and Supplementary information, Figure S2E). However, the expression of a number of early zygotic genes including BI891768, apoeb, lrwdl and soxlla, which are widely expressed in the whole blastoderm after MBT [47], was not obviously changed in net 1-MO1 injected embryos (Supplementary information, Figure S2F), suggesting an essential and specific function of net 1 in zebrafish dorsal fate specification. Furthermore, net 1 morphants showed a much smaller dorsal neuroectoderm (as indicated by sox 3 expression) which is normally induced by signals from the organizer, as well as an expanded ventral non-neural ectoderm (as indicated by gata 2 and $b m p 2 b$ expression; Figure $1 \mathrm{~F}$ and $1 \mathrm{G}$ ). To exclude the possibility that the decreased expression of dorsal marker genes in net 1 morphants is resulted from MO injection-induced global defects in cell proliferation or survival, we investigated whether there are differences in cell proliferation or apoptosis between $\mathrm{cMO}$ and MO1 injected embryos. Immunostaining for phosphorylated histone $\mathrm{H} 3$ indicated a similar vigorous cell proliferation in net 1 morphants compared with control embryos during gastrulation (Supplementary information, Figure S3A). In spite of the obvious cell apoptosis revealed by TUNEL assay in net1 morphants at $24 \mathrm{hpf}$, no apparent apoptosis signal was observed in either cMO- or MO1-injected embryos at gastrula stages (Supplementary information, Figure S3B). Consistently, p53 mutant embryos injected with 
A

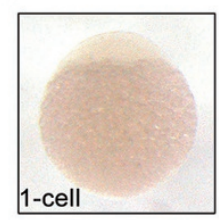

C

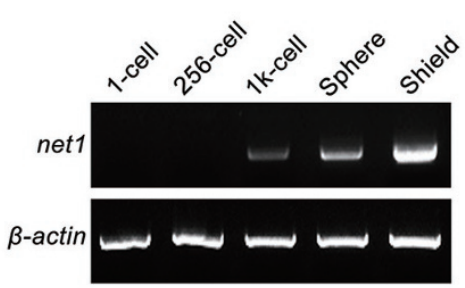

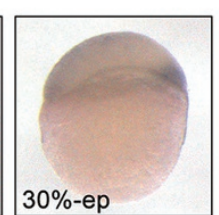

D

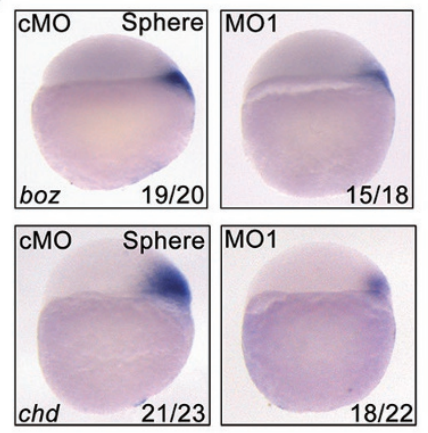

E

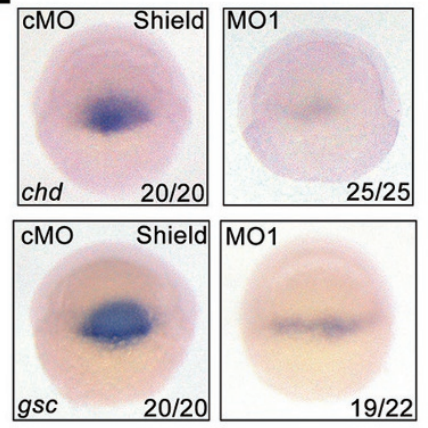

G

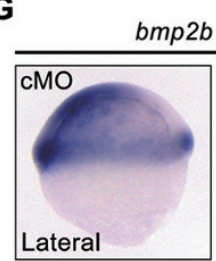

$b$ Shield
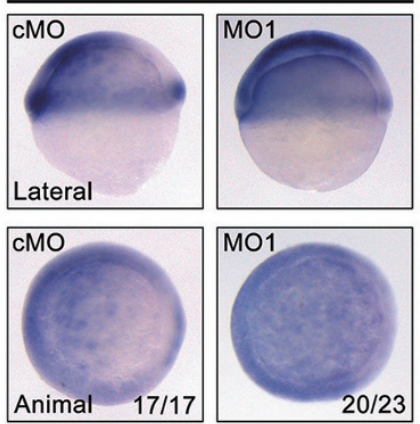

I'

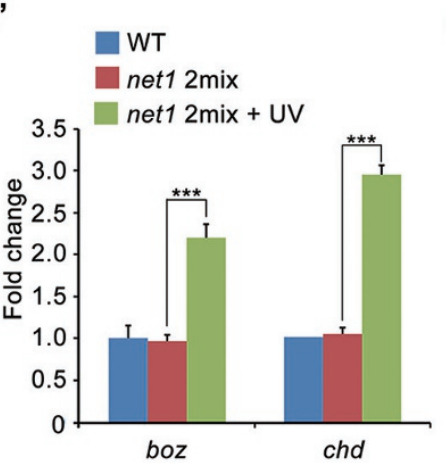

E'

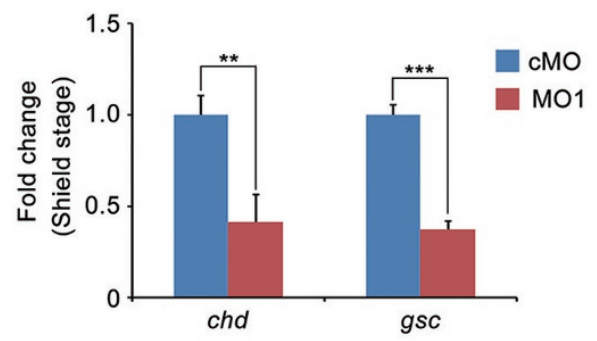

B

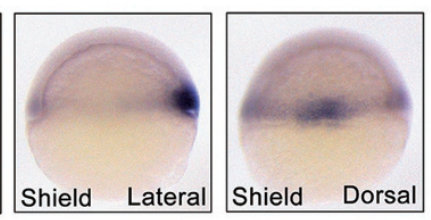

D'

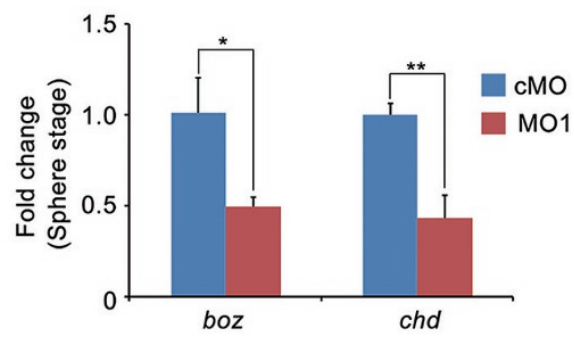

F

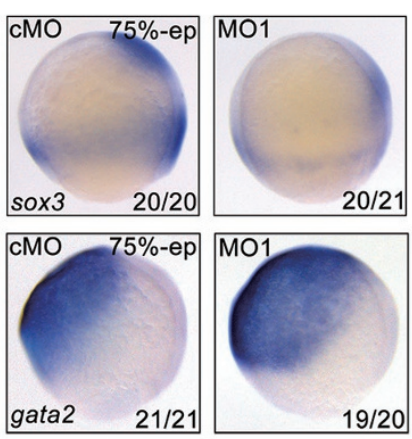

H

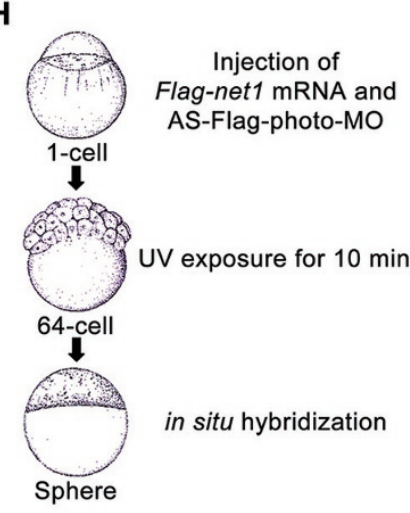

J

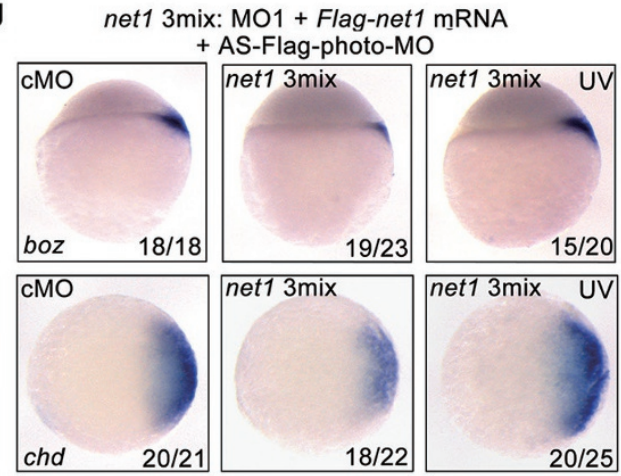

I net1 2mix: Flag-net1 mRNA + AS-Flag-photo-MO
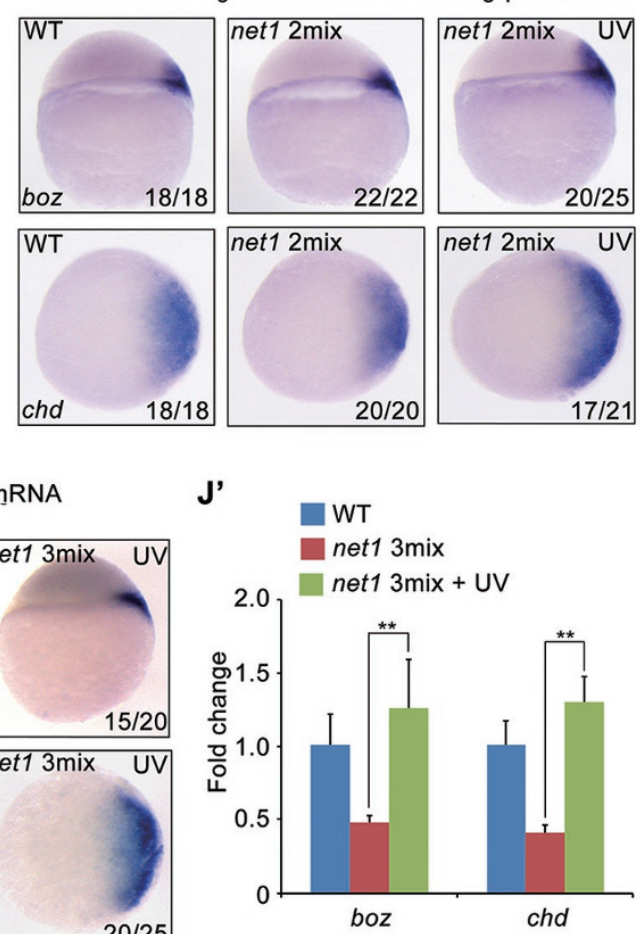
MO1 exhibited distinct morphological dorsal defects and decreased dorsal marker expression, further eliminating the potential confounding contribution of morpholino-induced $p 53$ activation and subsequent cell death (Supplementary information, Figure S3C and S3D). Furthermore, the expression of dorsal-related tissue marker genes, including prechordal plate marker gsc, forebrain marker six 3 and notochord marker $n t l$, was greatly decreased in net 1 morphants at bud and segmentation stages (Supplementary information, Figure S4A and S4B), suggesting that these dorsal defects are not caused by MO injection-induced developmental delay. To exclude potential off-target effects of MO injection, the siRNA-based gene-silencing strategy, which has been shown to be an alternative technique for knockdown of specific gene expression in zebrafish [48-50], was chosen to study the function of net 1 . We designed and synthesized two siRNAs (siRNA1 and siRNA2) targeting different sequences of zebrafish net 1 mRNA. Injection of either siRNA1 or siRNA2 into embryos dramatically decreased endogenous net 1 expression and resulted in dorsal defects at $24 \mathrm{hpf}$ (Supplementary information, Figure S5A and S5B). Further analyses revealed that dorsal fate determination was severely disrupted in net 1 siRNA-injected embryos (Supplementary information, Figure S5C). These observations suggest that net 1 is required for organizer formation and dorsal cell fate specification in zebrafish embryos.

Next we asked whether overexpression of net 1 could promote dorsal axis formation. As the injection of a very small amount of net 1 mRNA (10 pg) into 1-cell stage embryos led to severe malformations of the blastoderm and very early embryonic lethality, we used an antisense photo-cleavable morpholino targeting the $\mathrm{N}$-terminal
Flag sequence of Flag-net1 mRNA (AS-Flag-photo-MO) to block its early translation. Embryos were injected with a mixture containing Flag-net $1 \mathrm{mRNA}$ and AS-Flagphoto-MO (net1 2mix) at the 1-cell stage, exposed to UV at the 64-cell stage, then harvested at sphere stage for phenotype analyses (Figure 1H). Embryos overexpressing netl exhibited enlarged expression domains of dorsal markers boz and chd as compared with wild-type controls or embryos injected with net $12 \mathrm{mix}$ without UV exposure (Figure 1I and 1I'), indicating that net1 plays a positive role in dorsal axis formation. Importantly, the defects in dorsal fate specification in net 1 morphants could be rescued by overexpression of net 1 (Figure 1J and $1 \mathrm{~J}$ '), further suggesting the specificity of net 1 MOs. Likewise, the decreased expression of prechordal plate marker gsc and forebrain marker six 3 in bud stage morphants was also recovered by net 1 overexpression (Supplementary information, Figure S6). All these results show that net 1 acts as a zygotic factor to promote the formation of the early dorsal signaling center and establishment of the dorsal axis during zebrafish embryonic development.

\section{Net1 enhances Wnt/ $\beta$-catenin signaling}

Canonical Wnt signaling is essential for the formation of the dorsal organizer and establishment of the dorsal-ventral axis in vertebrate embryos [2, 4, 14]. The fact that net 1 is required for the specification of dorsal cell fates and expression of boz-the direct target of maternal Wnt/ $\beta$-catenin signaling [51], suggests that it might be an important regulator of $\mathrm{Wnt} / \beta$-catenin signal. To test our hypothesis, we first asked whether the expression of tbx 6 and $c d x 4$, two direct targets of zygotic Wnt/ $\beta$-catenin [52-54], are also regulated by net1. As shown in

Figure 1 Zebrafish Net1 is a positive modulator of dorsal axis formation. (A) Lateral views of net1 expression at indicated stages. (B) Western blot analysis of the temporal expression profile of Net1 protein in whole embryo lysates. Tubulin was used as loading control. (C) Semi-quantitative RT-PCR analysis of the net1 transcript profile at indicted stages. The expression of $\beta$-actin was used as an internal control. (D and E) The expression of dorsal marker genes boz, chd and gsc in cMO and net1 MO1-injected embryos at sphere stage and shield stage. (D) Lateral views with animal pole at the top. (E) Dorsal views with animal pole at the top. The ratios of affected embryos are indicated. (F) Analysis of the expression patterns of dorsal neuroectoderm marker gene sox 3 and ventral non-neural ectoderm marker gene gata2 at $75 \%$ epiboly stage. Lateral views with the dorsal side pointing to the right. $(\mathbf{G})$ The expression patterns of non-neural ectoderm marker gene $b m p 2 b$ in net1 morphants at shield stage. Lateral views with dorsal side to the right in upper panels; animal views with dorsal side to the right in lower panels. (H) Schematic diagram of the overexpression of net 1 in zebrafish. Embryos were injected with 200 pg Flag-net1 mRNA and 1 ng AS-Flag-photo-MO (net1 2mix) at one-cell stage then subjected to UV exposure at 64-cell stage for $10 \mathrm{~min}$. (I) The expression of boz and chd (animal views with dorsal side to the right) at sphere stage in embryos injected with net1 $2 \mathrm{mix}$. (J) Overexpression of net1 rescues the dorsal defects in net1 morphants. In all, 4 ng net1 MO1 together with 200 pg Flag-net1 mRNA and $1 \mathrm{ng}$ AS-Flag-photo-MO (net1 3mix) was co-injected into embryos, and a group of net1 $3 \mathrm{mix}$ injected embryos were exposed to UV. The expression of boz and chd was examined by in situ hybridization at sphere stage. (D', E', l' and J') The expression levels of indicated marker genes were individually examined by qRT-PCR. The expression levels of $\beta$-actin were used as a reference to normalize the amount of mRNAs in each sample. Asterisks indicate statistical significance of difference $\left({ }^{*} P<0.05,{ }^{* *} P<0.01,{ }^{* *} P<0.001 ; n=3\right)$. Error bars indicated SD. 
Figure 2A, at shield stage, tbx 6 and $c d x 4$ are specifically expressed in the ventrolateral mesoderm where net 1 is weakly expressed, and their expression was significantly reduced by injection of net1 MO1. Consistent with these results, knockdown of net 1 abrogated Wnt-induced expression of the $g f p$ reporter in the dorsal organizer and ventrolateral mesoderm of TOPdGFP transgenic embryos [55] (Figure 2B), indicating that net 1 is indispensable for both maternal and zygotic Wnt/ $\beta$-catenin signaling activity. Moreover, the decreased expression of dorsal marker genes boz and chd in net 1 morphants was restored to normal levels by co-injection with wnt $8 a$ or $\Delta N$ - $\beta$-catenin mRNA (Figure $2 \mathrm{C}$ ). These data suggest that zebrafish net 1 plays a pivotal role in maternal Wnt/ $\beta$-catenin signal transduction during early embryonic development. In addition, as zygotic $\mathrm{Wnt} / \beta$-catenin signal is required for the development of ventral tissues [56], the concomitant decrease of zygotic Wnt/ $\beta$-catenin signal activity in ventrolateral mesoderm in net 1 morphants was probably responsible for the absence of excessive tail tissues which are often observed in embryos with dorsal defects (Supplementary information, Figure S2C and S2D).

To identify whether net 1 is a feedback modifier of Wnt/ $\beta$-catenin signaling, expression of zebrafish net 1 was examined in embryos possessing either insufficient or excessive levels of Wnt. As shown in Supplementary information, Figure S7A, overexpression of wnt8a or $\triangle N$ - $\beta$-catenin mRNA (encoding a constitutively active form of $\beta$-catenin) was sufficient to induce ectopic expression of $c h d$, while inhibition of $\mathrm{Wnt} / \beta$-catenin signaling by injection of $\Delta N$-tcf 3 mRNA (encoding a dominant negative form of Tcf3) downregulated chd expression. Surprisingly, the expression of net 1 was not changed by either activation or inhibition of $\mathrm{Wnt} / \beta$-catenin signaling in zebrafish embryos (Supplementary information, Figure S7B).

We next investigated the role of Net1 in regulating the canonical Wnt pathway in mammalian cells. Overexpression of Net1 in HEK293T cells notably increased the Wnt3a-induced activity of the super-TOPFlash luciferase reporter in a dose-dependent manner (Figure 2D). To further explore the function of endogenous mammalian Net1, we analyzed the effect of net 1 knockdown on $\beta$-catenin/TCF-dependent transcription. We designed two short hairpin RNAs (shRNAs, named as netl shRNA1 and net1 shRNA2) against two different regions of human net 1, both of which effectively inhibited endogenous Net1 protein expression (Figure 2E). Transfection of HEK293T and MCF-7 cells with these shRNAs caused a significant decrease in luciferase activity compared with control (Figure 2F and 2G). This effect was further con- firmed by qRT-PCR analysis which revealed significantly reduced expression of $\mathrm{Wnt} / \beta$-catenin target genes Axin2, $c$-myc and LEF1 in net 1 -shRNA transfected HEK293T cells (Figure $2 \mathrm{H}$ ). Taken together, these results suggest that Net1 has a conserved function in potentiating Wnt/ $\beta$-catenin activity among different vertebrate species.

\section{Net1 requires $G E F$ activity to regulate $W n t / \beta$-catenin} signaling and dorsal axis formation

Net1 is a complex protein with a short C-terminal domain carrying a consensus PDZ-binding motif in addition to the catalytic DH domain and adjacent PH domain. A series of deletions and point mutations of Net1 were constructed and used to determine which domains were important for its subcellular localization and GEF activity $[34,36,57]$. To determine which domains of Net1 are important for $\mathrm{Wnt} / \beta$-catenin signaling, we generated a zebrafish deletion mutant named Net1- $\Delta C 4$, in which the last four amino acids carrying the PDZ-binding motif have been deleted [57], as well as two loss-of-function mutants denoted Net1-L266E and Net1-W437L, which contain point mutations in the $\mathrm{DH}$ and $\mathrm{PH}$ domains, respectively, resulting in loss of GEF activity [34]. HEK293T cells were transfected with these constructs in the presence or absence of Wnt3a to determine the effects on Wnt signaling using the super-TOPFlash luciferase reporter. We found that luciferase activity was dramatically increased by overexpression of both Net1$\triangle \mathrm{C} 4$ and wild-type Net1, suggesting that the PDZ-binding motif of Net1 is not necessary for the activation of Wnt/ $\beta$-catenin signaling (Figure 3A). In contrast, GEF mutants Net1-L266E and Net1-W437L were expressed at a similar level to wild-type Net1, but were no longer able to stimulate Wnt3a-induced luciferase activity (Figure 3B). Moreover, the reporter activity in net 1 shRNA1-transfected HEK293T cells was rescued by overexpression of wild-type zebrafish Net1, however, expression of either GEF mutant L266E or W437L did not (Figure 3C). In agreement with these results, co-injection of net $1 \mathrm{MO} 1$ with net $1-L 266 \mathrm{E}$ or $n e t 1-W 437 \mathrm{~L}$ mRNA, as compared with wild-type net $1 \mathrm{mRNA}$, could not rescue the decreased expression of dorsal markers boz and chd in net 1 morphants (Figure 3D). Furthermore, overexpression of wild-type net 1 but not net 1 GEF mutants expanded expression domains of chd and boz (Supplementary information, Figure S8). Based on these observations, we concluded that GEF activity is essential for Net1-mediated regulation of canonical Wnt signaling and dorsal fate specification.

Given that Net1 protein mainly localizes to the nucleus and that the translocation of Net1 into the cytoplasm is critical for its function in activating RhoA to stimu- 
A

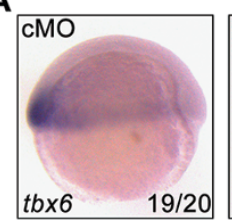

C
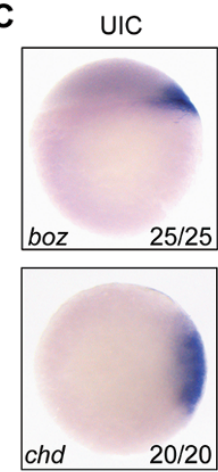

E
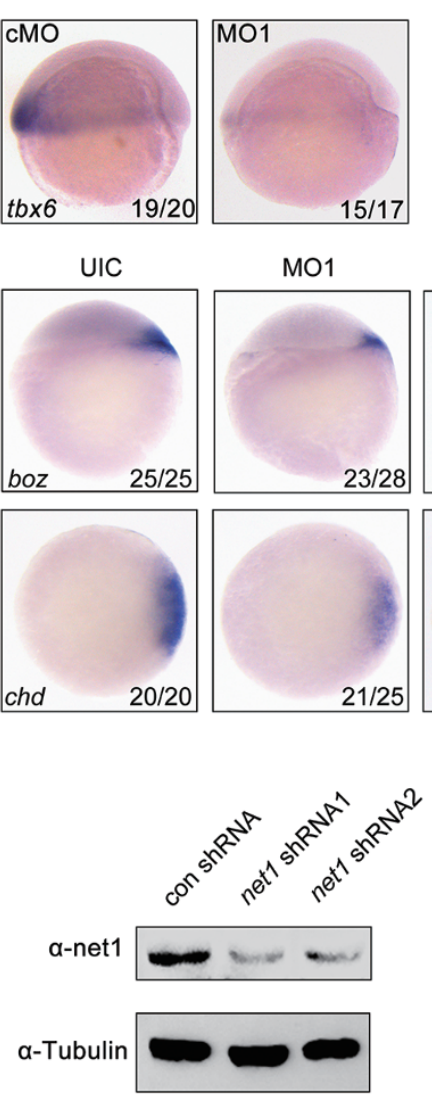

MO1
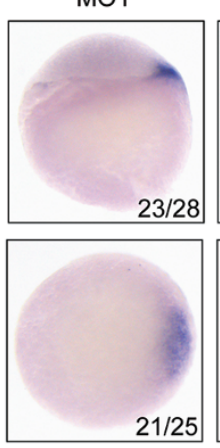

1/25

\section{F}

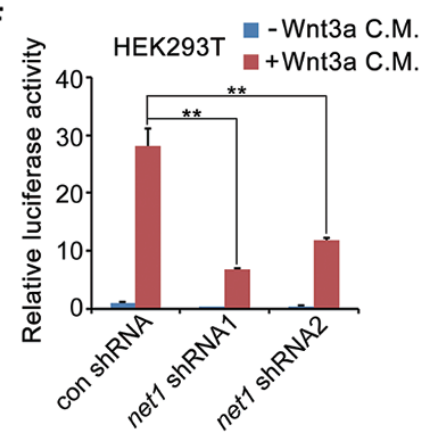

B

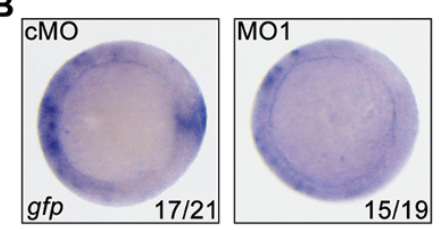

D

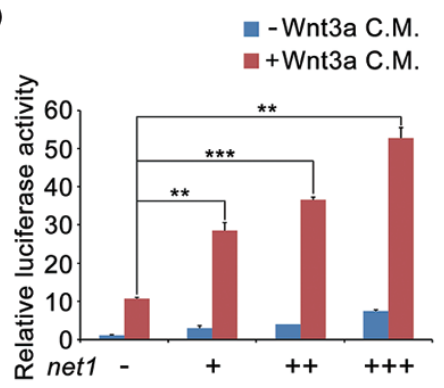

G

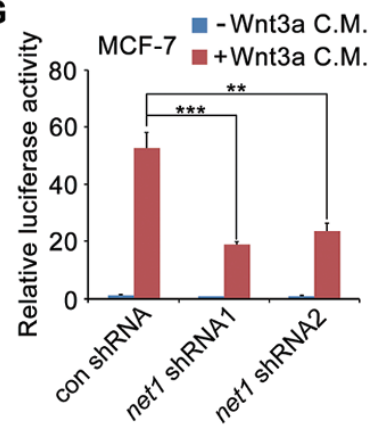

H
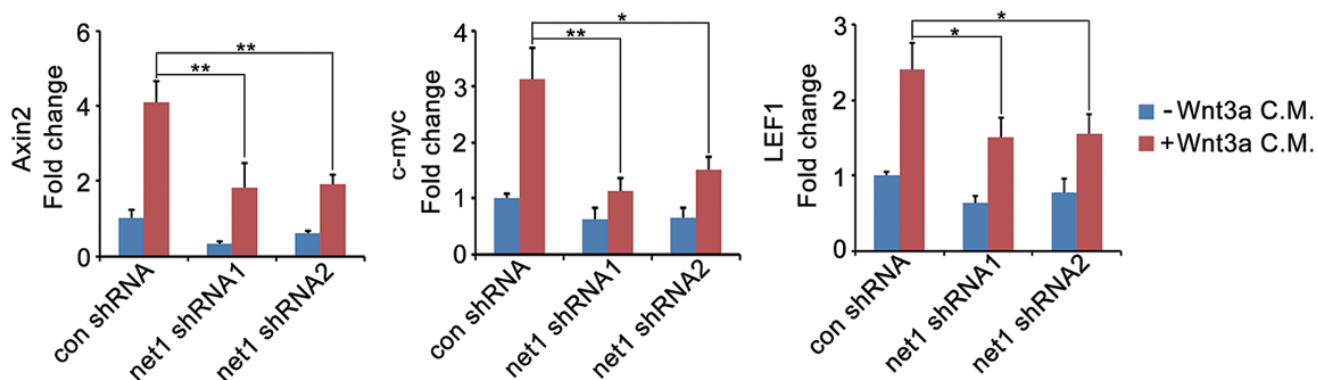

Figure 2 Net1 promotes Wnt/B-catenin signaling in zebrafish embryos and mammalian cells. (A) The expression of zygotic $\mathrm{Wnt} / \beta$-catenin target gene $t b x 6$ and $c d x 4$ in $4 \mathrm{ng} c M O$ or net1 MO1-injected embryos at shield stage. Lateral views with animal pole at the top. (B) Whole-mount in situ hybridization reveals the reduced expression of GFP in net1 MO1-injected TOPdGFP reporter transgenic zebrafish embryos. Embryos were injected with $4 \mathrm{ng} \mathrm{cMO}$ or net1 MO1 at the one-cell stage and harvested at shield stage for in situ hybridization. Animal views with dorsal side to the right. (C) Overexpression of wnt8a or $\Delta N-\beta$-catenin rescues dorsal development defects in net1 morphants. Embryos were injected with indicated MOs or mRNAs at the one-cell stage and harvested at shield stage for in situ hybridization with boz and chd probes. Injection doses: $\mathrm{cMO}$, 4 ng; net1 MO1, 4 ng; wnt8a mRNA, 10 pg; $\triangle N$ - $\beta$-catenin mRNA, 100 pg. (D) Overexpression of Net1 in HEK293T cells enhances super-TOPFlash luciferase activity in response to Wnt3a. HEK293T cells co-transfected with the super-TOPFlash luciferase reporter and increasing amounts of net1 expression plasmids were treated with or without Wnt3a CM for $12 \mathrm{~h}$, then harvested for luciferase assays. ${ }^{* *} P<0.01 ;{ }^{* * *} P<0.001$, Student's $t$-test. (E) net1 shRNA constructs efficiently knockdown endogenous human net1 expression. HEK293T cells were transfected with the indicated shRNA plasmids and harvested 48 $\mathrm{h}$ after transfection for western blot analyses. (F and G) net1 knockdown in HEK293T (F) and MCF-7 cells (G) decreases Wnt3a-induced super-TOPFlash luciferase activity. ${ }^{* *} P<0.01 ;{ }^{* *} P<0.001$, Student's $t$-test. (H) net1 knockdown in HEK293T cells inhibits the expression of Axin2, c-myc and LEF1. HEK293T cells transfected with the indicated plasmids were treated with or without Wnt3a CM for $12 \mathrm{~h}$, and harvested for qRT-PCR analysis. ${ }^{*} P<0.05$; ${ }^{*} P<0.01$, Student's $t$-test. 
late stress fiber formation $[35,58]$, we then investigated whether cytoplasmic distribution of Net1 is required to regulate $\mathrm{Wnt} / \beta$-catenin signaling. We generated zebrafish Net1 constructs in which the nuclear export signal (NES) sequence of MAPKK was added to the $\mathrm{N}$ terminus of an oncogenic version of Net1 ( $\Delta \mathrm{N}-\mathrm{Net}$, lacking the first 60 amino acids), and the NLS sequence of SV40 large T-antigen was added to the $\mathrm{N}$ terminus of wild-type Net1. As expected, NES- $\Delta$ N-Net1 was exclusively localized in the cytoplasm, whereas NLS-Net1 was restricted to the nucleus (Figure $3 \mathrm{E}$ ). In addition, GEF deficient mutations in NES- $\Delta$ N-Net 1 and NLS-Net1 did not change their subcellular localization (data not shown). Interestingly, either NES- $\Delta$ N-Net1 or NLS-Net1 significantly increased Wnt3a-induced luciferase reporter activity (Figure 3F), while their cognate GEF deficient mutants (L266E and W437L) did not (Figure 3G and 3H). Furthermore, overexpression of NES- $\triangle$ N-Net 1 or NLS-Net 1 recovered the ability of net 1-depleted cells to respond to Wnt3a stimulation (Figure 3I). Consistent with the results in mammalian cells, defects in dorsal axis formation in net 1 morphants were corrected by overexpression of $N E S-\triangle N$ net1 or NLS-net1 (Figure 3J). Taken together, these data suggest that both cytoplasmic and nuclear Net1 can promote canonical Wnt signaling and dorsal axis formation in a GEF activity-dependent manner.

Net1 controls phosphorylation of $\beta$-catenin at serine residue 675

To elucidate the mechanisms underlying net 1 function in dorsal development, we performed several experiments to map the position of Netl activity along the canonical Wnt signaling pathway. We have confirmed that Net1 is a key modulator of Wnt ligand-triggered signal activation (Figure $2 \mathrm{~F}$ and $2 \mathrm{G}$ ). We also found that shRNA knockdown of net 1 in HEK293T cells significantly attenuated expression of the super-TOPFlash reporter induced by GSK-3 $\beta$-selective inhibitors, $\mathrm{LiCl}$ and 6-bro-moindirubin-3-oxime (BIO) [59] (Supplementary information, Figure S9A and S9B). Moreover, Wnt3a-induced $\beta$-catenin nuclear accumulation was not changed in response to Net1 overexpression or depletion in mammalian cells (Supplementary information, Figure S9C-S9E). Likewise, knockdown of net1 in embryos did not result in obvious effects on the cellular distribution of endogenous $\beta$-catenin (Supplementary information, Figure S9F). These findings together suggest that Net1 acts in parallel with or downstream of $\beta$-catenin.

Phosphorylation of $\beta$-catenin at the C-terminal tyrosine and serine residues has been shown to increase its biological activity [21-24]. Specifically, the phosphorylation of S675 effectively modulates transcriptional activity of $\beta$-catenin in a variety of cells and tissues, but its effect on $\beta$-catenin stability is cell type-dependent $[22,23,60]$. We aimed to investigate whether $\beta$-catenin S675 phosphorylation is regulated by Net1 expression. In support of this hypothesis, overexpression of Net1 in HEK293T cells enhanced S675 phosphorylation in the presence of Wnt3a (Figure 4A). This increase in S675 phosphorylation relies on the presence of $\mathrm{Wnt} 3 \mathrm{a}$, as overexpression

Figure 3 Both cytoplasmic and nuclear Net1 regulate canonical Wnt signaling via its GEF activity. (A-C) Overexpression of wild-type zebrafish Net1 or Net1- $\Delta C 4$, but not its mutants without GEF activity, enhances Wnt/ $\beta$-catenin signal transduction (A and B) and restores the relative luciferase activity of super-TOPFlash reporters in net1-depleted HEK293T cells (C). HEK293T cells cotransfected with super-TOPFlash and the indicated plasmids were treated with or without Wnt3a CM for $12 \mathrm{~h}$, and harvested for luciferase assays. The expression level of wild-type Net1 and its various mutants was examined by western blots which were shown in the lower panels in A and B. ${ }^{* \star} P<0.01 ;{ }^{* * *} P<0.001$, Student's $t$-test. (D) Zebrafish embryos were co-injected with the indicated MOs and mRNAs at the one-cell stage and subjected to UV exposure as indicated. All embryos were harvested at sphere stage for in situ hybridization. net1 3mix, 4 ng net1 MO1 together with 200 pg Flag-net1 mRNA and 1 ng AS-Flag-photo-MO; L266E 3mix, 4 ng net1 MO1 together with 200 pg Flag-net1-L266E mRNA and 1 ng AS-Flag-photoMO; W437L 3mix, 4 ng net1 MO1 together with 200 pg Flag-net1-W437L mRNA and 1 ng AS-Flag-photo-MO. Note that the UV exposure-induced expression of wild-type Net1, but not its loss-of-function mutants, could restore the expression of dorsal marker genes in net1 morphants. (E) Hela cells were transfected with Flag-NES- $\Delta$ N-Net1 or Flag-NLS-Net1. Cells were fixed, immunostained with anti-Flag antibody and counterstained with DAPI to show the subcellular localization of these Net1 mutants. Scale bar, $10 \mu \mathrm{m}$. (F-I) HEK293T cells were transfected with the indicated plasmids and treated with or without Wnt3a C.M. before being harvested for luciferase assays. Note that the Wnt3a-induced response of super-TOPFlash reporter was enhanced by both cytoplasmic and nuclear Net1 (F). L266E and W437L mutants of the cytoplasmic and nuclear Net1, which lack GEF activity, lost the ability to promote Wnt/ $\beta$-catenin activity (G and $\mathbf{H}$ ). Either cytoplasmic or nuclear Net1and could restore the relative luciferase reporter activity in net1-depleted cells (I). ${ }^{*} P<0.05$; ${ }^{* *} P<0.01$; ${ }^{* *} P<0.001$, Student's $t$-test. (J) The dorsal developmental defects in net1 morphants are antagonized by cytoplasmic or nuclear Net1 overexpression. The expression of boz and chd were examined at the sphere stage in embryos injected with the indicated MOs and mRNAs. NES- $\Delta N$ 3mix, 4 ng net1 MO1 together with 200 pg Flag-NES- $\Delta N-n e t 1$ mRNA and 1 ng AS-Flag-photo-MO; NLS 3mix, 4 ng net1 MO1 together with 200 pg Flag-NLS-net1 mRNA and 1 ng AS-Flag-photo-MO. 
A

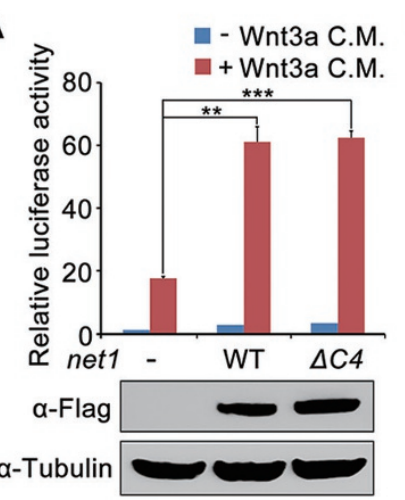

D
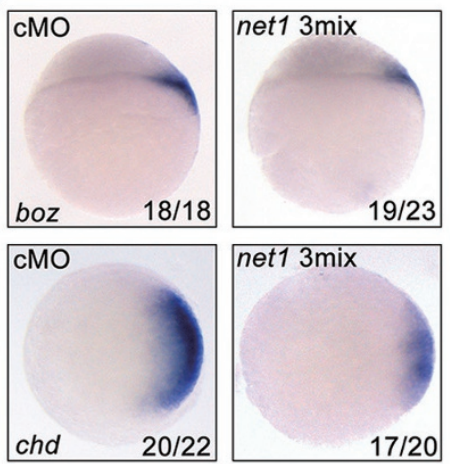

$\mathbf{F}$

F $\square$ - Wnt3a C.M. $\square+$ Wnt3a C.M. $\mathbf{G}$
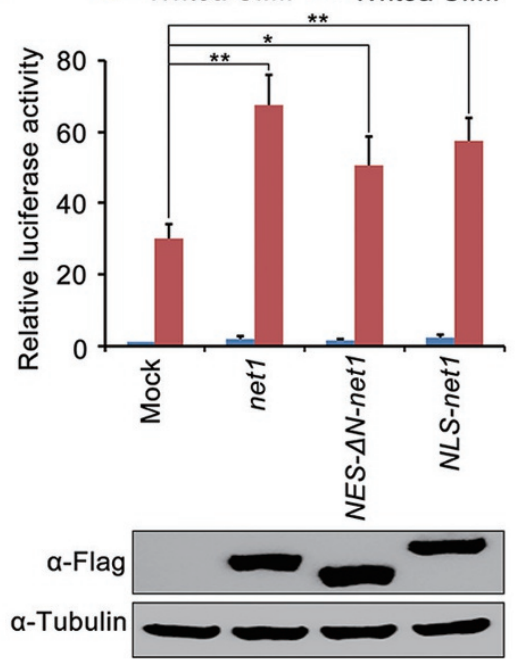

I $\square-$ Wnt3a C.M. $\square+$ Wnt3a C.M.

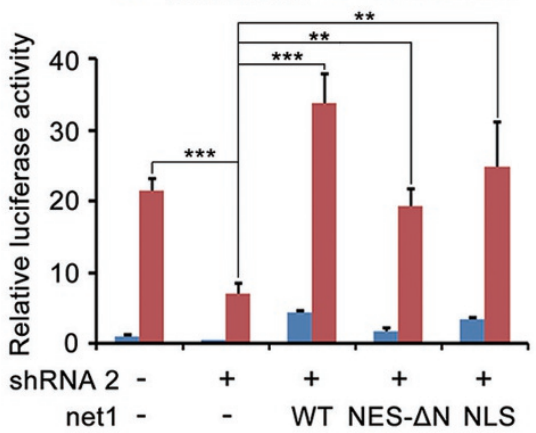

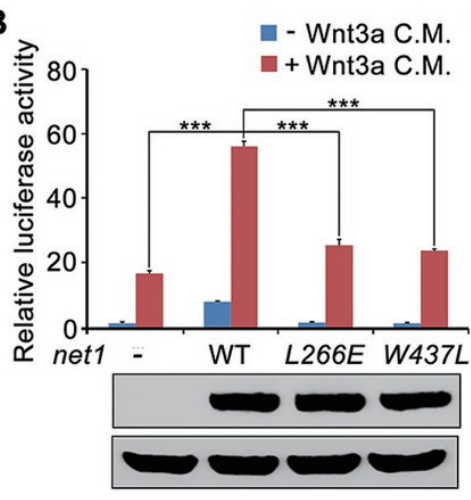
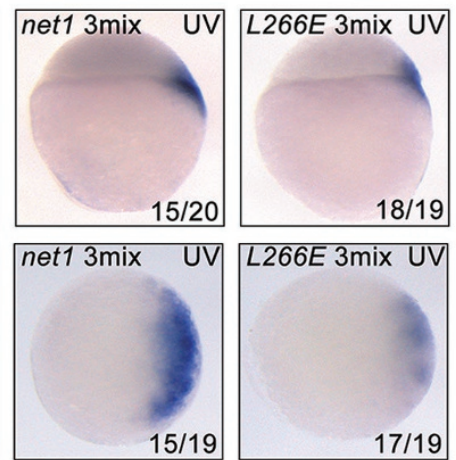

\section{C}

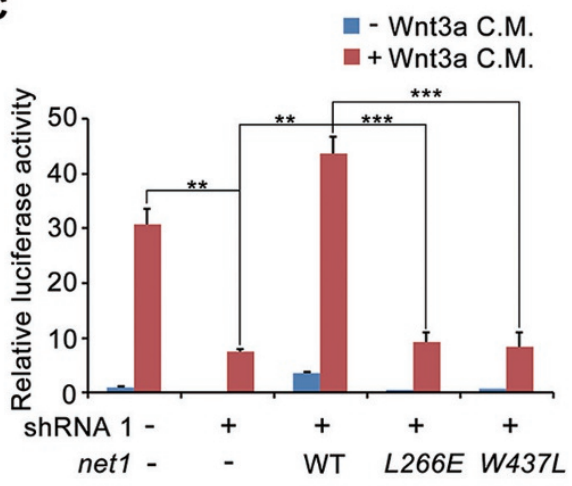

E

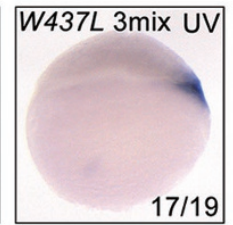

L266E 3mix UV W437L 3mix UV
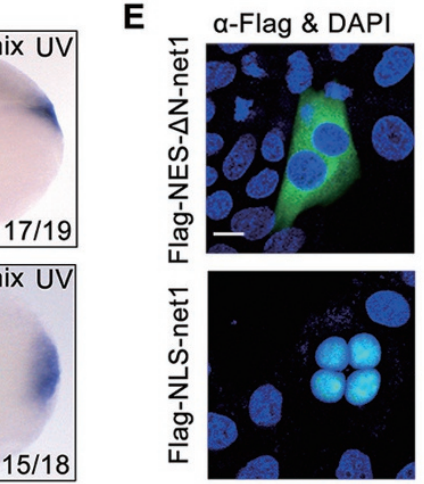

\section{G}

- Wnt3a C.M. $=+$ Wnt3a C.M.

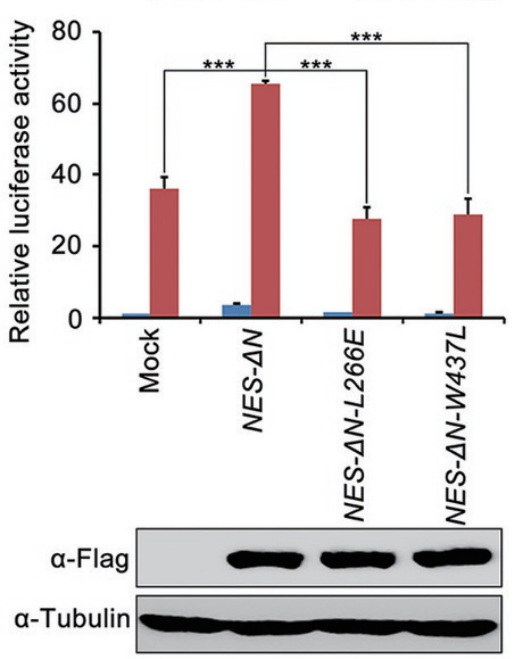

$15 / 18$

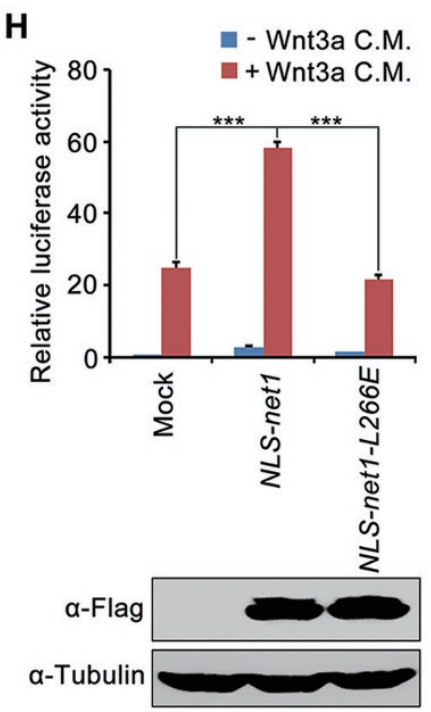

\section{J}
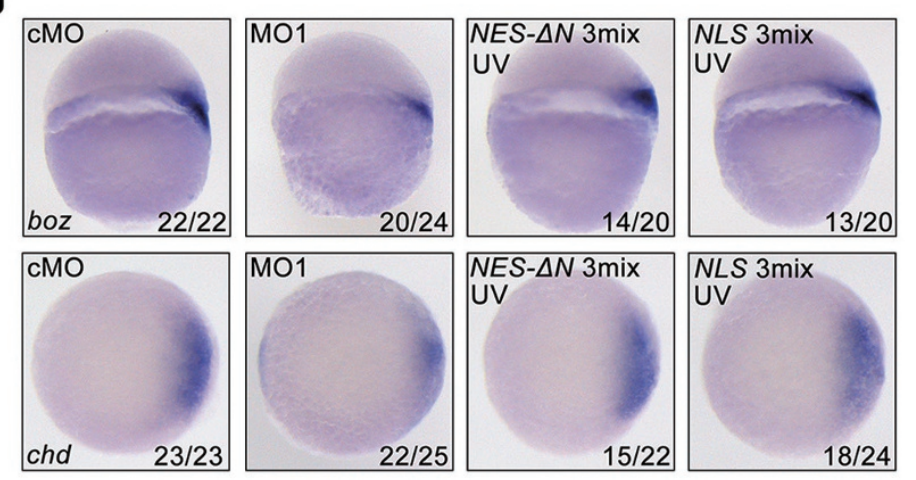
of Net1 alone could not induce S675 phosphorylation (Figure 4A). In addition, Wnt3a stimulation significantly promoted wild-type $\beta$-catenin phosphorylation which could not be detected in S675A mutant-the unphosphorylated form of $\beta$-catenin (Supplementary information, Figure S10A). These data demonstrate the specificity of the anti-phospho- $\beta$-catenin (Ser675) antibody we used in this study and further confirms the requirement of Wnt signal activation in $\beta$-catenin S675 phosphorylation.

Consistent with the requirement of Net1 GEF activity in regulating canonical Wnt signal, overexpression of Net1-L266E or Net1-W437L mutants did not increase the phosphorylation level of $\beta$-catenin S675 in the presence of Wnt3a (Figure 4B). We further tested the role of endogenous Net1 in regulating $\beta$-catenin phosphorylation. Knockdown of net1 expression in HEK293T cells resulted in a significant decrease of basal and Wnt3a-induced phosphorylation levels of $\beta$-catenin S675 (Figure 4C). Reintroduction of wild-type Net1, but not its GEF defective mutants, into net 1 -depleted cells reversed the reduction of $\beta$-catenin S675 phosphorylation (Figure 4D). Thus, Net1 contributes to $\beta$-catenin S675 phosphorylation.

We subsequently addressed the biological functions of Net1-regulated $\beta$-catenin S675 phosphorylation during zebrafish dorsal axis formation. Endogenous $\beta$-catenin in zebrafish embryos was phosphorylated at $\mathrm{S} 675$ at a very low level before the $1 \mathrm{~K}$-cell stage, but this phosphorylation significantly increased during the MBT (Figure 4E). Phosphorylation of $\beta$-catenin S675 was almost abolished in net1 morphants compared with wild-type control (Fig- ure $4 \mathrm{E}$ ), suggesting that net 1 is the major positive modulator of $\beta$-catenin S675 phosphorylation during MBT. Injection of $200 \mathrm{pg}$ mRNA of wild-type net1, but not its loss-of-function mutants, could restore phosphorylation level of $\beta$-catenin at S675 in net1 MO1-injected embryos (Figure 4F), indicating the requirement for Net1 GEF activity for $\beta$-catenin S675 phosphorylation. To verify the functional relevance of S675 phosphorylation in dorsal axis formation, we overexpressed $\beta$-catenin S675D and S675A mutants, which respectively mimic phosphorylated and unphosphorylated forms of the protein and retain the same cellular localization as the wild-type [23]. In situ hybridization assays revealed that only injection of $\beta$-catenin $S 675 D$ mRNA could significantly expand the expression domain of boz and chd in wild-type embryos (Figure 4G). Furthermore, $\beta$-catenin $S 675 D$ mRNA also recovered the expression defects of these dorsal marker genes in net 1 morphants (Figure 4G). Injection of $\beta$-catenin $S 675 D$ mRNA induced much more expanded expression domains of boz and chd compared with wildtype $\beta$-catenin overexpression, and most importantly, resulted in a much earlier expression of these dorsal marker genes at 512-cell stage or high stage when their transcripts could not be detected in wild-type embryos or wild-type $\beta$-catenin mRNA-injected embryos (Figure $4 \mathrm{H}$ and 4I). Taken together, $\beta$-catenin S675 phosphorylation is required for dorsal gene expression, and net 1 reinforces dorsal development by increasing the phosphorylation of $\beta$-catenin at residue S675.

Since Nodal signal is require for the pre-dorsal organizer expression of net1, we next examined whether the

\footnotetext{
Figure 4 Net1-regulated $\beta$-catenin S675 phosphorylation is required for zebrafish dorsal axis formation. (A and B) Overexpression of wild-type Net1 (A) but not Net1 loss-of-function mutants (B) results in an increase in $\beta$-catenin S675 phosphorylation. HEK293T cells transfected with plasmids encoding Flag-Net1, Flag-Net1-L266E or Flag-Net1-W437L were treated with or without Wnt3a CM. Lysates were analyzed by western blot using the indicated antibodies. (C) Knockdown of net1 leads to a significant decrease in $\beta$-catenin S675 phosphorylation. HEK293T cells were transfected with net1 shRNA expressing plasmids and treated with or without Wnt3a CM before immunoblotting. (D) HEK293T cells were co-transfected net1shRNA and Net1 plasmids, then treated with Wnt3a CM. Total cell lysates were prepared and immunoblotted with the indicated antibodies. Note that only wild-type Net1 can rescue the relative decrease in $\beta$-catenin $\mathbf{S 6 7 5}$ phosphorylation in net1-depleted cells. (E and F) Western blots of total lysates from wild-type embryos, 4 ng cMO or net1 MO1-injected embryos, and net1 morphants co-injected with 200 pg mRNAs encoding wild-type net1 or net1 mutants. The phosphorylation level of $\beta$-catenin S675 is upregulated around MBT (1 K-cell stage) in wild-type embryos (E, left panels), while this relative increase in $\beta$-catenin S675 phosphorylation is abolished in net1 morphants (E, right panels). $\beta$-cat, $\beta$-catenin. Only overexpression of wild-type Net1 can rescue the decrease in $\beta$-catenin $\mathbf{S} 675$ phosphorylation in net1 morphants (F). In panel A-F, quantification is the relative density of phospho-specific signals to corresponding total protein signals (mean \pm SD, three independent biological repeats). (G) The phosphorylation of $\beta$-catenin $\mathrm{S} 675$ is essential for dorsal gene expression. The expression of boz and chd was examined at the sphere stage in embryos injected with the indicated MOs and mRNAs. Injection dose: $\beta$-catenin S675D mRNA, 150 pg; $\beta$-catenin S675A mRNA, 400 pg; net1 MO1, 4 ng. (H) Phosphorylated $\beta$-catenin has stronger ability to enhance dorsal gene expression. The expression of boz and chd was examined at the sphere stage in embryos injected with the $150 \mathrm{pg}$ indicated mRNAs. (I) Phosphorylated $\beta$-catenin induces much earlier expression of boz and chd. The expression of boz and chd was examined at the 512-cells stage and High stage, respectively, in embryos injected with 150 pg indicated mRNAs.
} 
A

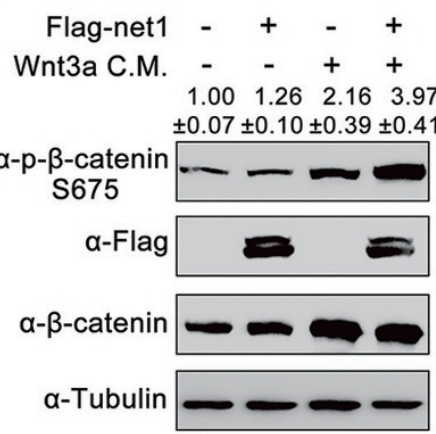

D

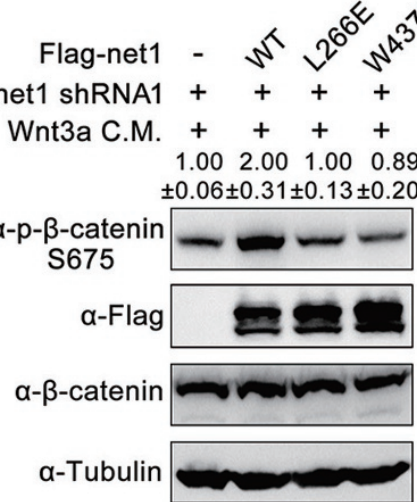

B

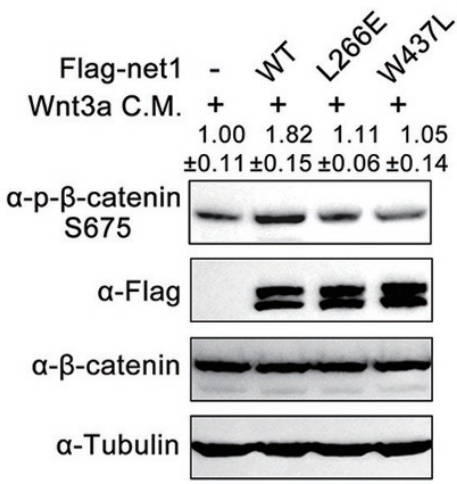

C

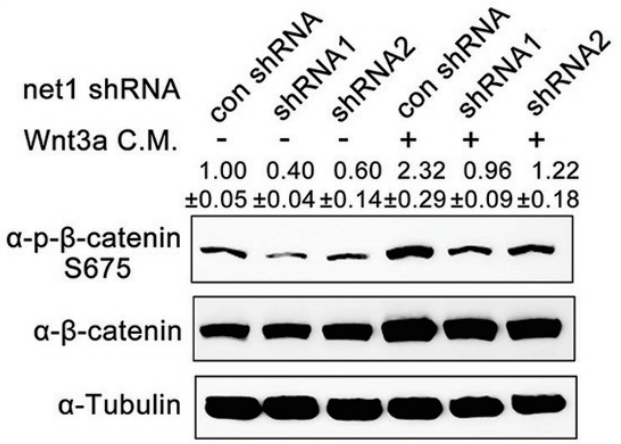

$E$

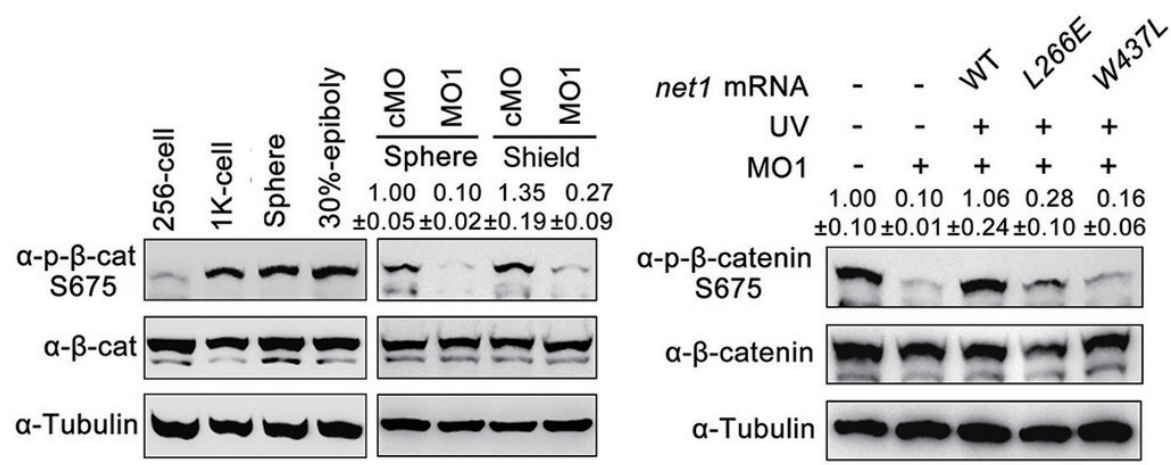

G
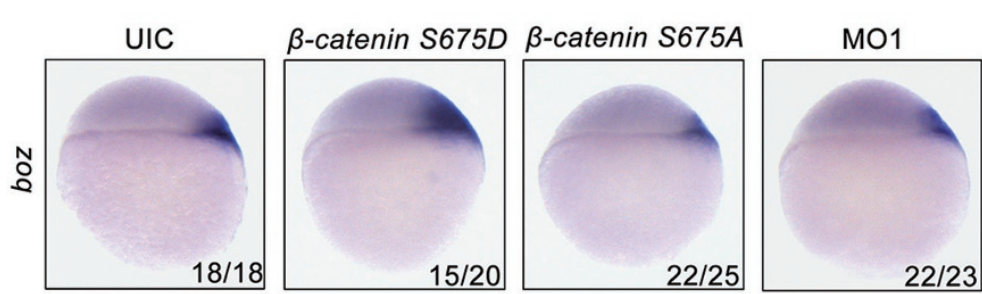

$\mathrm{MO} 1+$

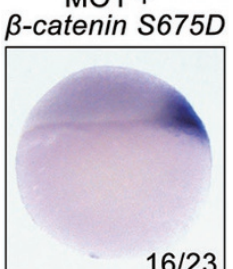

$\mathrm{MO} 1+$
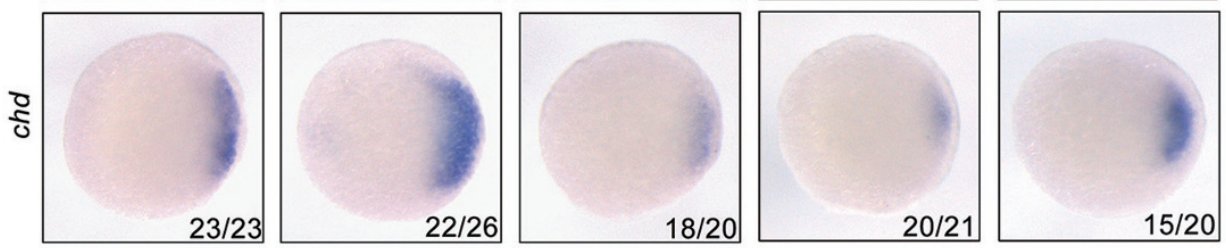

$25 / 27$

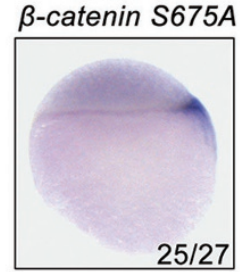

H

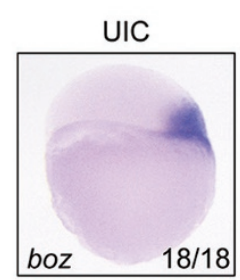

$\beta$-catenin WT
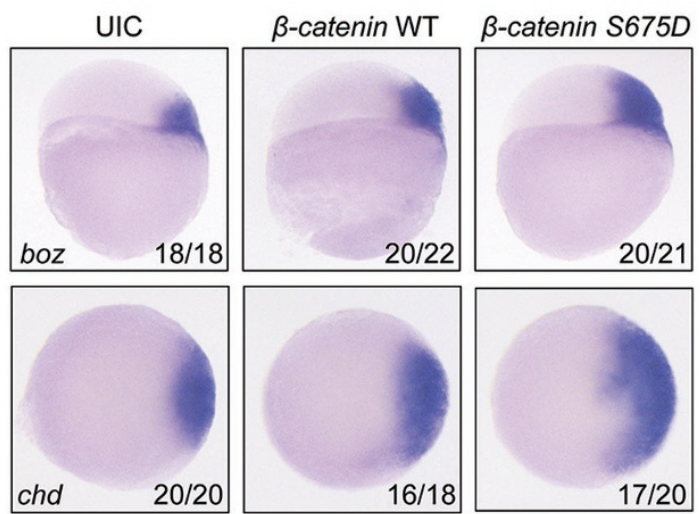

$20 / 22$

$20 / 21$
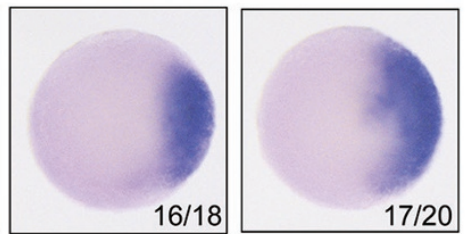

I
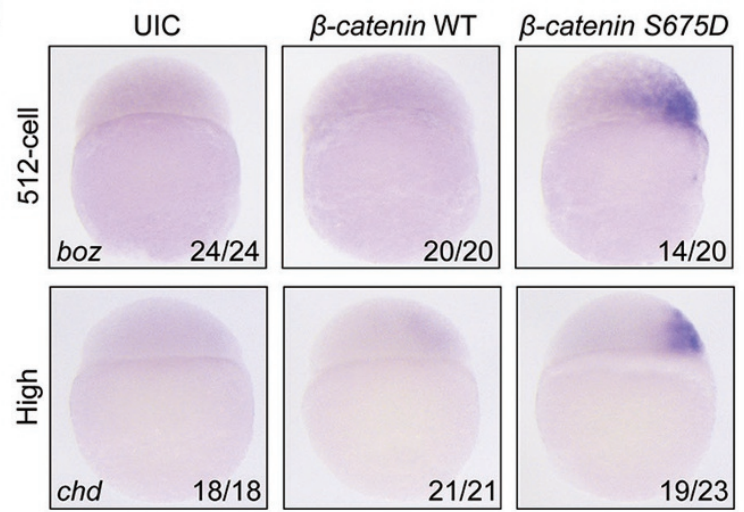
$\beta$-catenin S675 phosphorylation is decreased in Nodal-deficient embryos. Interestingly, MZoep mutants exhibited similar phosphorylation level of $\beta$-catenin S675 as is in wild-type embryos at sphere and $30 \%$ epiboly stages (Supplementary information, Figure S10B). As Smad4-deficient HCT116 cells show elevated PAK1 expression [61], we speculate that the expression of some Nodal-suppressed genes might be upregulated to maintain $\beta$-catenin phosphorylation in MZoep mutants.

Net1 acts upstream of PAK1 to regulate $\beta$-catenin phosphorylation

Since $\mathrm{S} 675$ of $\beta$-catenin has been shown to be a major target of PAK1 and protein kinase A (PKA) [22, 23], we first examined the potential involvement of these two kinases during dorsal axis formation in zebrafish. Embryos injected with the mRNA encoding a specific inhibitory protein for PKA (PKI) $[22,62]$ or treated with the PKA selective chemical inhibitor H89 [63] exhibited normal expression of the dorsal markers and the phospho- $\beta$-catenin (S675; Supplementary information, Figure S11A and S11B), thus excluding a major role for PKA in zebrafish dorsalization. These results are in good agreement with previous reports that the level of PKA activity in the dorsal region is lower than that in the ventral region from late blastula to gastrula stages, and PKA modulates morphogenetic movement but not cell fate specification during Xenopus gastrulation [64]. However, when embryos were treated with IPA-3, a selective non-ATP competitive PAK1 inhibitor, or injected with pak1 K299A mRNA, a dominant negative PAK1 mutant (dnpak1) [23], expression of dorsal markers and phospho- $\beta$-cat- enin (S675) was dramatically reduced (Figure 5A and Supplementary information, Figure S11C). Furthermore, overexpression of pakl T423E, a constitutively active form of PAK1 (capak1) [23], expanded the dorsal marker expression domains in wild-type embryos, and also rescued the phenotypes resulting from net1-depletion in morphants (Figure 5A and 5B). To explore whether the activity of PAK1 is differentially activated in the dorsal and ventral regions of the embryos after MBT, gsc:GFP transgenic fish embryos were injected with pak1 mRNA (encoding Flag-PAK1) at 1-cell stage, and cut into dorsal and ventral halves with a microblade at $30 \%$ epiboly stage. Expression of GFP, and phosphorylation of PAK1 Thr423 which occurs in activated PAK1 [65], were only detected in the dorsal regions of zebrafish embryos, indicating that embryos were correctly dissected into ventral and dorsal halves and PAK1 is primarily activated in the dorsal blastomeres (Figure 5C). Importantly, net1 MO1 injection strongly repressed PAK1 phosphorylation in zebrafish embryos (Figure 5C). Likewise, injection of the mRNA of capak1, but not dnpak1, into net1 morphants derepressed the phosphorylation of $\beta$-catenin S675 (Figure 5D). Thus, PAK1 activity appears to be required for dorsal axis formation, and Net1 likely promotes the $\mathrm{C}$-terminal phosphorylation of $\beta$-catenin by activating PAK1 in the dorsal blastomeres.

We next examined the epistatic relationship between Net1 and PAK1 in modulating canonical Wnt signaling. Overexpression of CAPAK1 increased the luciferase activity of the super-TOPFlash reporter by approximately threefold over the control in response to Wnt3a stimulation, whereas DNPAK1 caused an approximately 50\%

Figure 5 Net1 controls $\beta$-catenin S675 phosphorylation via PAK1. (A) Expression patterns of boz and chd in sphere stage embryos treated with $10 \mu \mathrm{M}$ IPA-3 or embryos injected with 225 pg dnpak1 or 300 pg capak1 mRNA. (B) Expression patterns of boz and chd in sphere stage embryos co-injected with $4 \mathrm{ng}$ net1 MO1 and $300 \mathrm{pg}$ capak1 mRNA. Note that overexpression of capak1 restored the expression of dorsal marker genes in net1 morphants. (C) net1 is essential for PAK1 activition in dorsal region. gsc:GFP transgenic embryos injected with 200 pg Flag-PAK1 mRNA and indicated MOs were cut into dorsal and ventral halves under fluorescence microscope. The dissected tissues (left panel) or whole embryos (right panel) were lysed with TNE at $30 \%$ epiboly stage. Then lysates were subjected to immunoprecipitation and western blot analysis with the indicated antibodies. (D) Western blots of total lysates from sphere-stage embryos injected with the indicated MOs and mRNAs. net1 MO1, 4 ng; capak1 mRNA, 300 pg; dnpak1 mRNA, 225 pg. (E-G) HEK293T cells were transfected with the indicated plasmids and treated with or without Wnt3a CM before being harvested for luciferase assays. The Wnt3a-induced response of the super-TOPFlash reporter was enhanced by CAPAK1 and inhibited by DNPAK1 (E). Forced expression of DNPAK1 inhibited the ability of net1 to enhance Wnt3a-induced luciferase activity (F), but overexpression of CAPAK1 significantly increased Wnt/ $\beta$-catenin signal transduction in net1-depleted cells (G). NS, non significant; ${ }^{*} P<0.05 ;{ }^{* *} P<0.01$; ${ }^{* * *} P$ $<0.001$, Student's $t$-test. (H) Western blot analysis showing reduced p-PAK1 in HEK293T cells transfected with plasmids expressing net1 shRNA1 or shRNA2 for $48 \mathrm{~h}$. (I-K) Cell lysates from HEK293T cells co-transfected with the indicated plasmids were subjected to immunoprecipitation with anti-Flag M2 agarose beads. Total lysates and IPs were subjected to western blot analysis with the indicated antibodies. Note that overexpression of wild-type Net1, but not its loss-of-function mutants, strongly blocked the interaction between $\beta$-catenin and HDAC1 (I). In D and I, quantification is the relative density of phospho-specific signals to corresponding total protein signals, while in $\mathbf{H}$, quantification is the mean relative ratio of co-immunoprecipitated signals over lysate (mean $\pm \mathrm{SD}$, three independent biological repeats). 
A

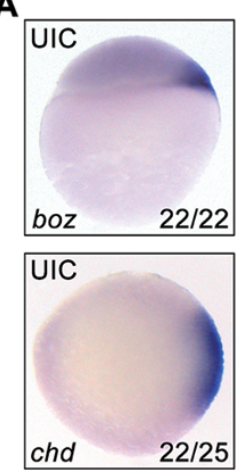

C

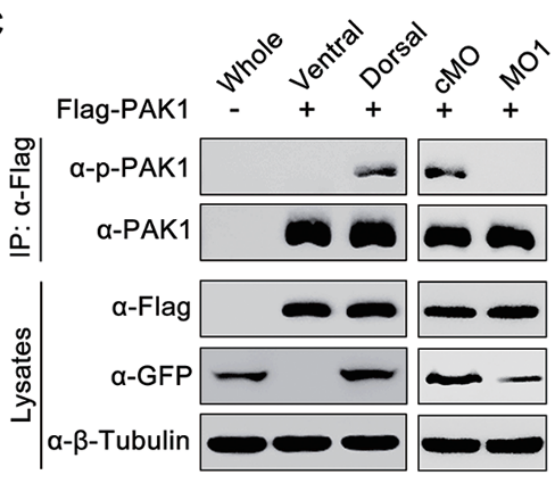

$\mathbf{F}$

a -Wnt3a C.M. $\quad+$ +Wnt3a C.M.

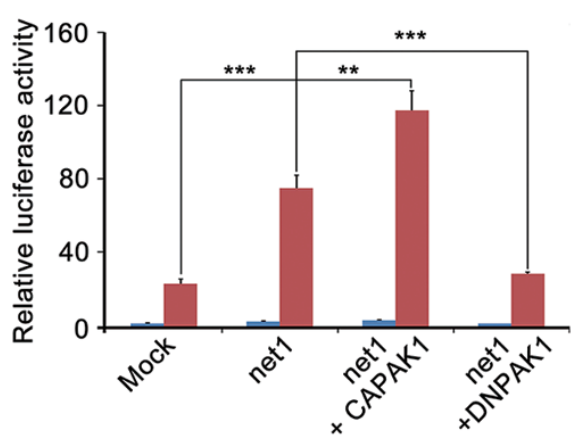

I

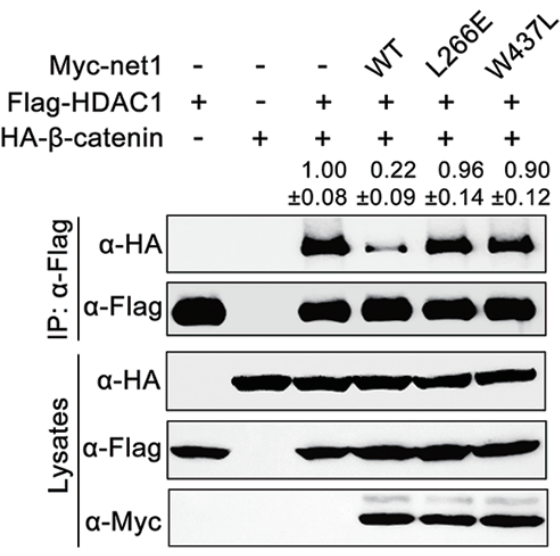

D

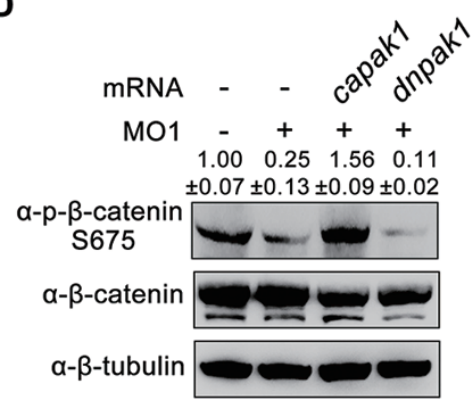

E
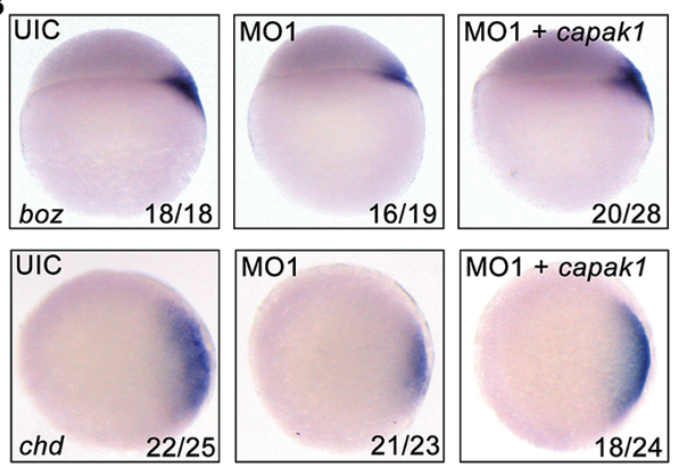

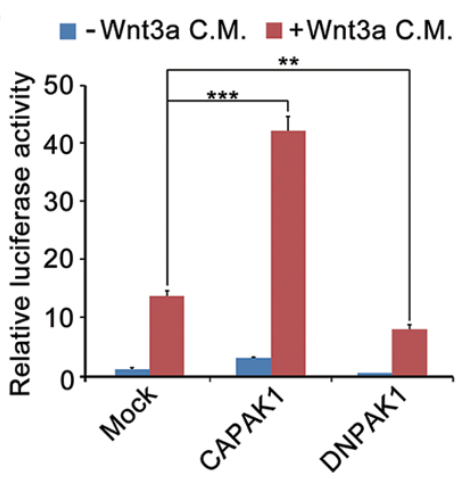

G

- Wnt3a C.M. $\quad+$ +Wnt3a C.M.

H
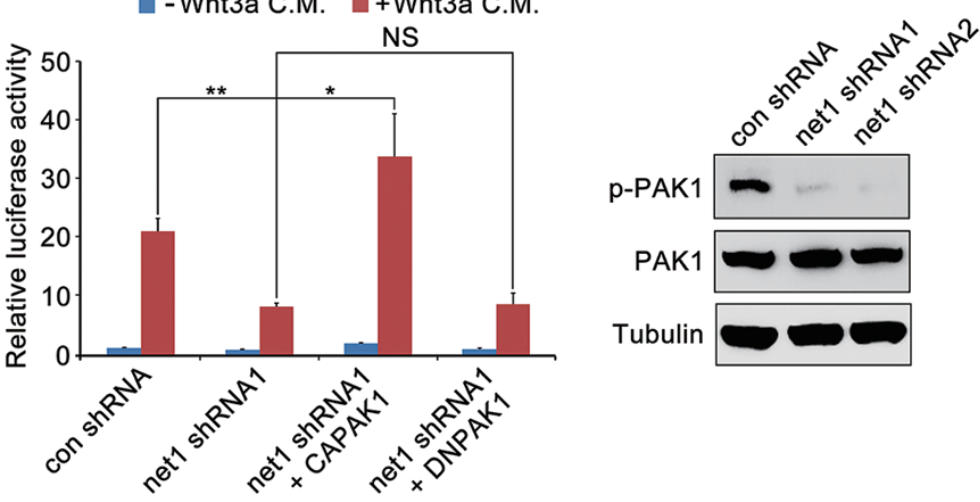

$\mathbf{K}$

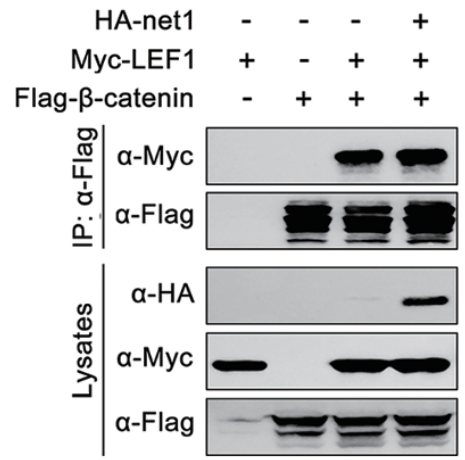


decrease in reporter activity (Figure 5E), suggesting that PAK1 is a positive regulator of canonical Wnt signaling as previously reported [23]. Importantly, co-expression of CAPAK1 with Net1 further increased luciferase reporter activity, while disruption of endogenous PAK1 by forced expression of DNPAK1 inhibited the ability of Net1 to enhance Wnt3a-induced luciferase activity (Figure 5F). However, overexpression of CAPAK1, but not DNPAK1, still dramatically induced $\mathrm{Wnt} / \beta$-catenin signal transduction in net1-depleted cells (Figure 5G). In addition, the expression of phosphorylated PAK 1 (Thr423) was abolished in net 1 shRNA transfected cells (Figure $5 \mathrm{H}$ ). PAK1 has been reported to promote $\beta$-catenin transcriptional activity by inhibiting the association of $\beta$-catenin and its general transcriptional repressor HDAC1 $[23,66]$. Consistent with these previous reports, wild-type Net1, but not its GEF mutants, could strongly block $\beta$-catenin/HDAC1 interaction, but had no effects on $\beta$-catenin/LEF1/TCF 4 association (Figure $5 \mathrm{I}-5 \mathrm{~K}$ ). These results suggest that Net1 acts upstream of PAK1 to regulate $\beta$-catenin phosphorylation and ultimately Wnt-responsive gene transcription.

\section{Net1 dissociates and activates PAK1 dimers to promote $\beta$-catenin phosphorylation}

After establishing the functional significance of the Net1-PAK 1 cascade in promoting $\beta$-catenin activation during zebrafish dorsal development, we aimed to elucidate the mechanism by which Net1 enhances PAK1 activation in mammalian cells and zebrafish embryos. Co-immunoprecipitation experiments were first performed to examine relative binding affinity. The results indicated that Myc-tagged or endogenous PAK1 was spe- cifically co-precipitated with overexpressed Flag-Net1 in HEK293T cells (Figure 6A and 6B). The amount of Myc-PAK1 co-immunoprecipitated with GEF defective mutants, Flag-Net1-L266E or W437L, was significantly decreased compared with wild-type control (Figure 6C), suggesting that their association requires Net1 GEF activity. Moreover, in contrast with wild-type PAK1, the H83L/H86L mutant containing an inactive GTPase binding domain (GBD), which cannot bind small GTPases such as Cdc42 and Rac1 [65], showed a much lower affinity for Net1 (Figure 6D). Thus, Net1 is a binding partner of PAK1, and the interaction of these two proteins requires Netl GEF activity.

Previous studies have shown that majority of PAK1 exists as inactive homodimers by the interaction between the N-terminal inhibitory switch domain and the C-terminal kinase domain [67]. GTP-bound Cdc42 or Rac1 binds to GBD of PAK1 disrupting its dimerization, resulting in fully autophosphorylated and activated monomers [65]. Both the decreased phosphorylation level of PAK1 Thr423 in net 1-depleted cells (Figure 5H) and the physical association between these two proteins prompted us to examine whether Net1 controls PAK1 dimerization. HEK293T cells were transfected with plasmids expressing Flag-PAK1 and Myc-PAK1. Myc-PAK1 could be specifically detected in anti-Flag immunoprecipitates, suggesting the overexpressed PAK1 is in dimerized form (Figure 6E). As expected, PAK1 homodimers were dissociated upon co-expression of wild-type Net1 but not its mutants without GEF activity (Figure 6E and 6F). Interestingly, dimers of DNPAK1, the kinase-inactive mutant K299A, was similarly disrupted by Net1 overexpression (Figure 6G). This finding is consistent with the previous

Figure 6 Effect of Net1 on PAK1 dimerization. (A-D) Flag-tagged Net1 interacts with overexpressed (A) or endogenous (B) PAK1. HEK293T cells were transfected as indicated with expression plasmids encoding Myc-tagged PAK1 and Flag-tagged Net1 or Net1 mutants, then harvested for immunoprecipitation with anti-Flag M2 agarose beads. Note that compared with the wild-type control, Flag-Net1-L266E or W437L has a much lower binding affinity for Myc-PAK1 (C). Conversely, Myc-tagged PAK1 H83L/H86L mutant (which cannot bind Rho family small G proteins such as Cdc42 and Rac1) loses the ability to associate with wild-type Net1 (D). (E and F) Wild-type Net1 (E) but not its L266E or W437L mutant (F) disrupts dimerization of PAK1. HEK293T cells were co-transfected with plasmids expressing Flag- or Myc-tagged PAK1 and HA-tagged wild-type Net1 or Net1 mutants as indicated. Lysates were immunoprecipitated with anti-Flag M2 agarose beads and immunoblotted as indicated. (G) Net1 disrupts the dimerization of DNPAK1, the kinase-inactive mutant. HEK293T cells were co-transfected with plasmids expressing Flag- or Myc-tagged PAK1 K299A mutant proteins and HA-tagged Net1. Lysates were subjected to immunoprecipitations and immunoblots. (H) net1-depletion enhances PAK1 dimerization. HEK293T cells transfected with net1 shRNA1 or shRNA2 were harvested for immunoprecipitation and immunoblotting. (I and $\mathbf{J})$ Wnt3a CM treatment enhances the interaction between PAK1 and Net1, and disassociates the dimerization of PAK1. HEK293T cells co-transfected with indicated plasmids were treated with or without Wnt3a C.M. for $12 \mathrm{~h}$ before harvest, and then lysed for immunoprecipitations. ( $K$ and $\mathbf{L}$ ) Net1 enhances the binding affinity of PAK1 for $\beta$-catenin (K) and promotes PAK1-induced phosphorylation of $\beta$-catenin S675 (L) in the presence of Wnt3a. HEK293T cells transfected with the indicated constructs were treated with or without Wnt3a CM for $12 \mathrm{~h}$ before harvesting for immunoprecipitation and western blot analyses. In $\mathbf{C}-\mathbf{K}$, quantification is the mean relative ratio of co-immunoprecipitated signals over lysate, while in $\mathbf{L}$, quantification is the relative density of phospho-specific signals to corresponding total protein signals (mean $\pm \mathrm{SD}$, three independent biological repeats). 
A

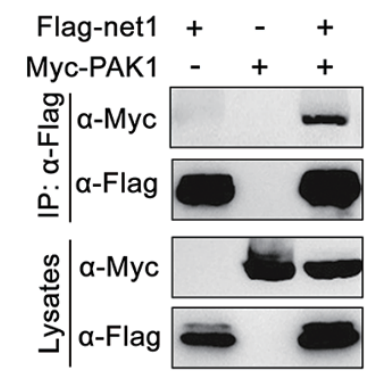

D

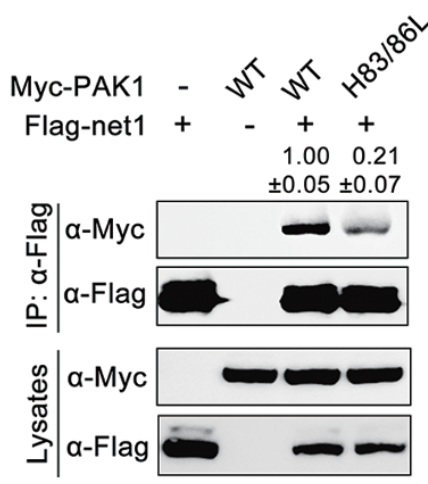

G

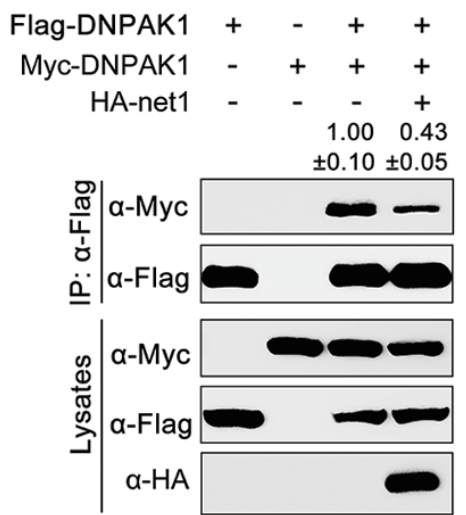

$\mathbf{J}$

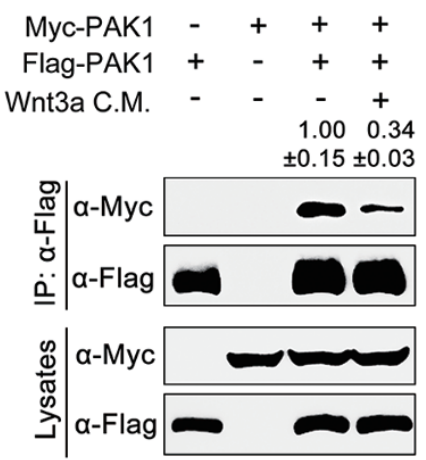

B

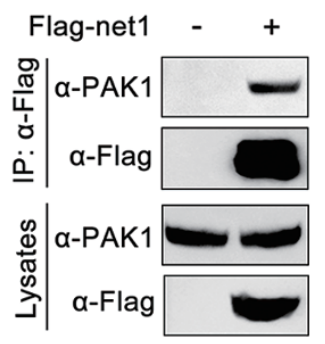

E

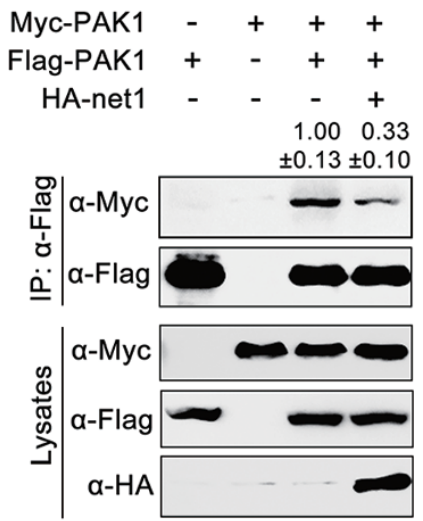

H

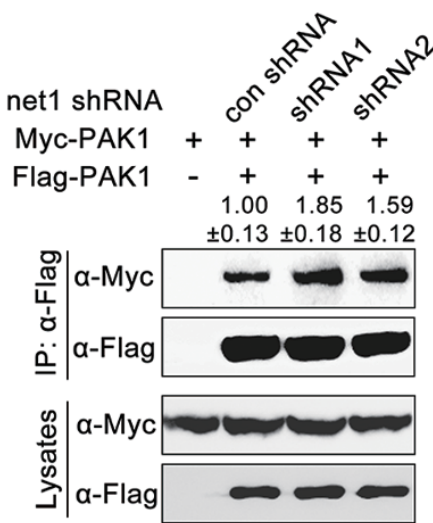

K

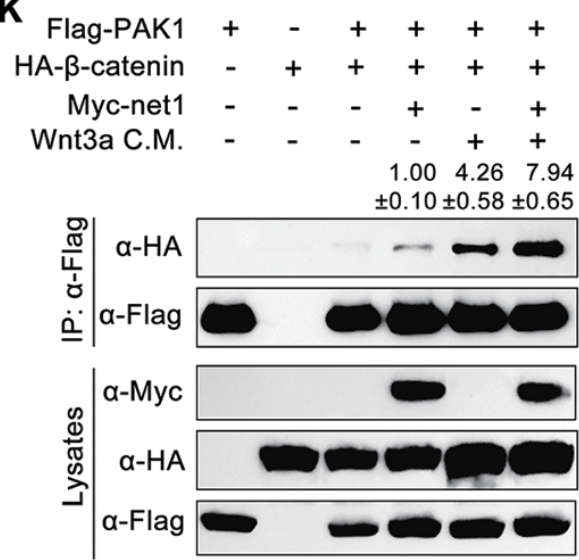

C

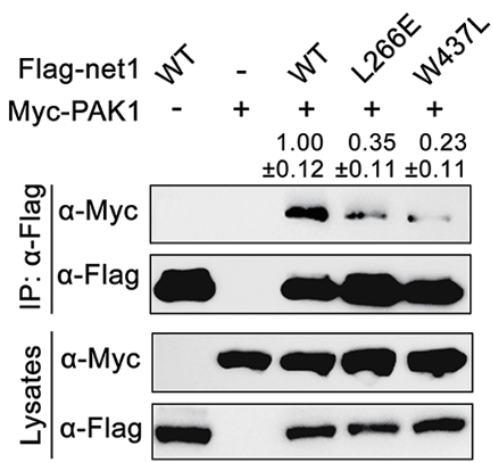

$\mathbf{F}$

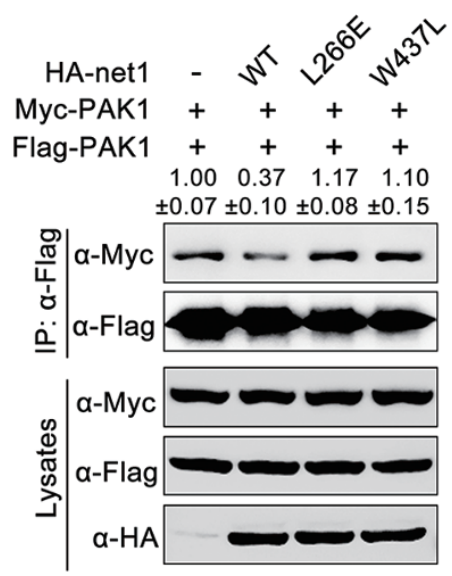

I

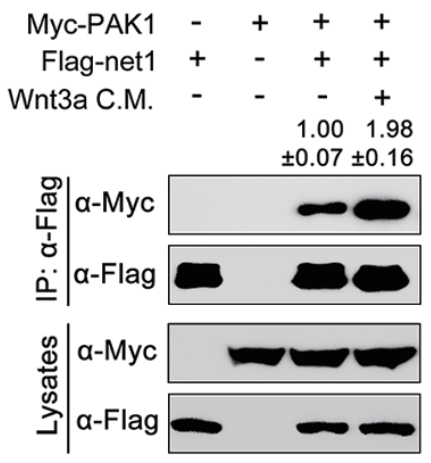

$\mathbf{L}$

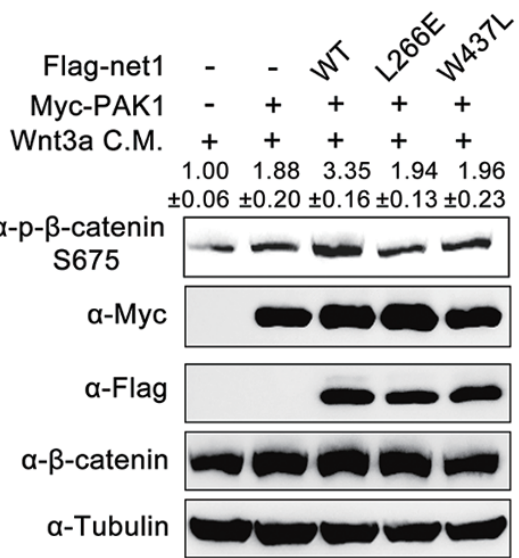


conclusions that PAK1 kinase-dead mutant, which lost its ability to autophosphorylate the crucial Thr423 residue, efficiently forms homodimers and its dimerization is strongly dissociated upon GTPase binding [65]. These data also suggest that the disruption of PAK1 homodimer by Net1 is not a consequence of autophosphorylation of key residues, including Thr423. In contrast, the association of Flag-PAK1 with Myc-tagged PAK1 was moderately enhanced in net 1 shRNAs transfected cells (Figure $6 \mathrm{H})$. These results reveal that Net1 and its GEF activity are essential for the dissociation of PAK1 homodimers.

In addition, Wnt3a stimulation strengthened the association of Net1 with PAK1 and promoted the dissociation of PAK1 homodimers (Figure 6I and 6J). Co-expression of Net1 with PAK1 enhanced the affinity of PAK1 kinase for its substrate $\beta$-catenin in the presence of Wnt3a (Figure 6K). Consistently, PAK1-induced phosphorylation of $\beta$-catenin S675 was increased in cells transfected with plasmids expressing wild-type Net1 but not Net1 mutants (Figure 6L). These results therefore support the idea that Net1 promotes $\beta$-catenin phosphorylation via disrupting PAK1 dimerization.

Net1 activates an unidentified Rho family GTPase to regulate Wnt signaling and dorsal development

The requirements of Net 1 GEF activity during Net1-regulated PAK1 activation and $\beta$-catenin phosphorylation suggest the involvement of GTPases in these processes. Although mammalian Net1 specifically activates RhoA but not Rac1 or Cdc42 [34, 68], whether Net1 functions on zebrafish RhoA, Rac1 and Cdc42 activation has not been investigated. Previous work demonstrated that the GBD of PAK1 (PBD) binds the activated GTPbound forms of Rac1 and Cdc42, but not RhoA, while the GBD of Rhotekin (RBD) selectively binds the activated GTP-bound form of RhoA, but not Rac1 or Cdc42 [69-71]. GST-PBD and GST-RBD fusion proteins were generated and used as probes in pull-down assays to test the activation of these GTPases by Net1 overexpression in zebrafish embryos. Consistent with their mammalian homologous proteins, zebrafish Rac1, Cdc42 and RhoA were able to bind GST-PBD and GST-RBD, respectively (Supplementary information, Figure S12A). Overexpression of zebrafish Net1 significantly increased the binding of GST-RBD and zebrafish RhoA, but had no effect on the binding of GST-PBD and zebrafish Rac1 or Cdc42, suggesting Net1 selectively activates zebrafish RhoA (Supplementary information, Figure S12A). RhoA has context-dependent functions either enhancing or restraining $\mathrm{Wnt} / \beta$-catenin signal $[72,73]$; we then examined whether RhoA was involved in dorsal development. Injection of RhoA-specific inhibitor C3 or overexpression of the dominant-negative rhoA mutant (rhoA-N19) in zebrafish embryos resulted in significant morphological changes including reduced body size and shortened anterior-posterior body axis at $24 \mathrm{hpf}$ (Supplementary information, Figure S12B and S12C). Consistently, in rhoA-N19 mRNA injected embryos, the adaxial mosodermal tissues exhibited shorter anterior-posterior axis with more distant bilateral columns (as indicated by myodl expression), and the notochord became wider and shorter (as indicated by $n t l$ expression; Supplementary information, Figure S12D). These phenotypes resembled the CE movement defects observed in rhoA morphants [41]. The inhibition of RhoA activity did not cause obvious dorsal fate defects during early embryonic development (Supplementary information, Figure S12E and S12F). Thus, RhoA is not necessary for dorsal fate specification.

To confirm the involvement of GTPases in Net1-induced $\beta$-catenin activation and dorsal development, we used compactin, a pan Rho family GTPase inhibitor, to reduce endogenous GTPase activity in mammalian cells and zebrafish embryos [74]. In HEK293T cells, compactin treatment dramatically reduced the association between Net1 and PAK1 (Figure 7A), and further inhibited the ability of Net1 to disrupt PAK1 dimerization (Figure 7B). As expected, ectopic Net1 expression stimulated super-TOPFlash reporter activity (Figure 7C). However, the Net1-induced promotion of reporter expression was significantly repressed by compactin treatment (Figure 7C). Importantly, compactin treatment decreased the expression of dorsal marker genes in wild-type embryos, and erased the rescue effects of net 1 mRNA on dorsal axis formation in net 1 morphants (Figure 7D and 7E). These data provide evidence that an unidentified Rho family small GTPase is required for Net1-regulated PAK1 homodimer dissociation, canonical Wnt signal transduction and dorsal axis formation. Overall, these results support a model consisting of the GEF Net1, which activates an unidentified GTPase but not RhoA to bind to the GBD domain of PAK1. Net1 then dissociates PAK1 homodimers and relieves the inhibition of its kinase activity to enhance canonical Wnt signal transduction by phosphorylating $\beta$-catenin at S675 during dorsal development of ebrafish embryo (Figure 7F).

To further confirm the function of net 1 in dorsal axis formation, we generated net 1 mutants using Cas9/gRNA system targeting exon 2, which encodes a unique amino acid sequence upstream of the DH domain [75]. We obtained two deletion mutants named net1420 (20 bp deletion with $1 \mathrm{bp}$ insertion) and net1453 (53 bp deletion) (Supplementary information, Figure S13A). Both of these two net 1 mutations led to a shift of the open-reading frame with a premature stop codon. The results of in 
situ hybridization experiments with net 1 probe and western blot analyses using an anti-Net1 antibody showed that the expression of net 1 transcript and protein was totally abolished in net $1 \Delta 20$ and net1 $\Delta 53$ homozygous embryos (Supplementary information, Figure S13B and S13C), suggesting that these two mutants are null alleles of the net 1 gene. At first glance, net1 120 and net $1 \Delta 53$ homozygotes showed no obvious morphological defects compared with wild-type control (Supplementary information, Figure S13D). Further analyses revealed that the dorsal marker genes were normally expressed at early stages during embryo development (Supplementary information, Figure S13E). Thus, unlike the severe dorsal developmental defects observed in net 1 morphants, no obvious phenotypes are found in net 1 mutants.

The phenotypic differences between mutants and morphants may be due to the activation of a compensatory network in mutants $[76,77]$. Actually, net $1 \mathrm{MO} 1$ or siRNA1 injection caused dorsal fate defects in wild-type embryos, but did not influence the dorsal development of mutants (Supplementary information, Figure S13F and S13G), indicating the specificity of the MOs and siRNAs used to knock-down zebrafish net 1 in our study and the insensitiveness of the net 1 mutants to net $1 \mathrm{MOs}$ and siRNAs. To identify the molecules with a compensatory role in net 1 mutants, we performed RNA sequencing analyses in wild-type embryos, net1 morphants and net1 153 homozygotes at sphere stage. We identified a set of genes encoding GEFs, GTPases, and GTPase effectors that were upregulated in net1 $\Delta 53$ mutants but not in wildtype embryos and net1 morphants (Figure 7G). Quantitative real-time PCR experiments using a number of prim- ers listed in Supplementary information, Data S1, further confirmed the specific activation of most of these genes in net $1 \Delta 53$ mutants (Figure $7 \mathrm{H}$ ). Furthermore, net $1 \Delta 53$ mutants treated with compactin or injected with dnpakl mRNA exhibited clearly decreased expression of dorsal markers, suggesting that the upregulation of these GTPase-mediated signal transduction-related genes in net 1 mutants compensates for the loss of netl (Supplementary information, Figure S13H and S13I). In addition, these results provide valuable evidence from another perspective to support the important function of Net1 and its GEF activity in activating $\mathrm{Wnt} / \beta$-catenin signaling during dorsal development.

\section{Discussion}

The neuroepithelial cell transforming gene net 1 encodes a RhoA-specific GEF that regulates cell migration in different embryonic stages of various animal species [38-40]. In this study, we found that zebrafish net1 is prominently expressed in the dorsal side of the embryo after MBT. Loss-of-function and gain-of-function experiments in zebrafish embryos and mammalian cells revealed that Net1 acts as a zygotic factor to trigger dorsal regionalization. Mechanistically, Net1 disrupts PAK1 dimerization by activating an unknown GTPase, and subsequently promotes PAK1-mediated phosphorylation of $\beta$-catenin at $\mathrm{S} 675$ to fully activate $\mathrm{Wnt} / \beta$-catenin signaling during the dorsal development of zebrafish embryos. To our knowledge, this is the first genetic evidence to demonstrate that Net1 contributes to dorsal axis formation by modulating maternal Wnt signaling.

Figure 7 An unidentified Rho family GTPase is necessary for Net1-regulated canonical Wnt signal transduction and dorsal axis formation. (A-C) Compactin treatment inhibits the interaction between Net1 and PAK1 (A), the Net1-induced dissociation of PAK1 homodimers (B) and Net1-promoted increase in super-TOPFlash reporter luciferase activity (C). HEK293T cells were treated with or without $10 \mu \mathrm{M}$ compactin for $6 \mathrm{~h}$ then transfected with the indicated plasmids. After transfection, cells were subjected to compactin treatment for another $24 \mathrm{~h}$ before harvesting for immunoprecipitation and western blot analyses $\left(\mathbf{A}\right.$ and $\mathbf{B}$ ) or luciferase assays $(\mathbf{C})$. ${ }^{* * *} P<0.001$, Student's $t$-test. In $\mathbf{A}$ and $\mathbf{B}$, quantification is the mean relative ratio of co-immunoprecipitated signals over lysate (mean \pm SD, three independent biological repeats). (D) Compactin treatment disrupts the dorsal development of wild-type embryos. Wild-type embryos were treated with $10 \mu \mathrm{M}$ compactin from 16-cell stage until sphere stage, before harvesting for in situ hybridization. (E) Compactin treatment suppresses the rescue effects of net1 mRNA on dorsal axis formation in net1 morphants. Embryos were co-injected with the indicated MOs and mRNAs at the one cell stage. net1 3 mix injected-embryos were subjected to compactin treatment and UV exposure as indicated. All embryos were harvested at sphere stage for in situ hybridization. net1 3mix: 4 ng net1 MO1 together with 200 pg Flag-net1 mRNA and 1 ng AS-Flag-photo-MO. (F) An integrated model for the activation of canonical Wnt signaling by Net1. U-GDP, GDP-bound form of unidentified GTPase; U-GTP, GTP-bound form of unidentified GTPase. (G and H) A number of Rho family GTPase signal transduction-related genes are upregulated in net1 mutants compared with wild-type embryos and net1 morphants. (G) Heat map of 16 Rho family GTPase signal transduction-related genes which are differentially expressed in wild-type embryos, net1 morphants and net1 $\Delta 53$ mutants. The transcript abundance was quantified from RNA-Seq data. Induced genes are represented in red, while suppressed genes are represented in green. $(\mathbf{H})$ The expression differences of these 16 genes in wild-type embryos, net1 morphants and net1 $\Delta 53$ mutants were validated by qRT-PCR analyses. ${ }^{*} P<0.05 ;{ }^{* *} P<0.01 ;{ }^{* * *} P<$ 0.001 , Student's $t$-test. 
A

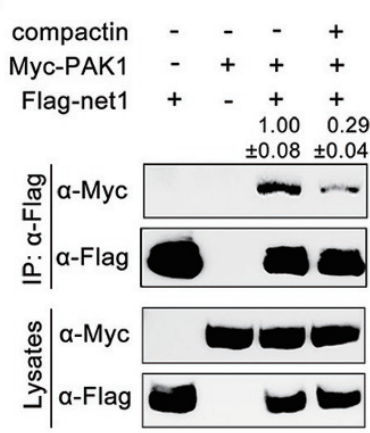

D
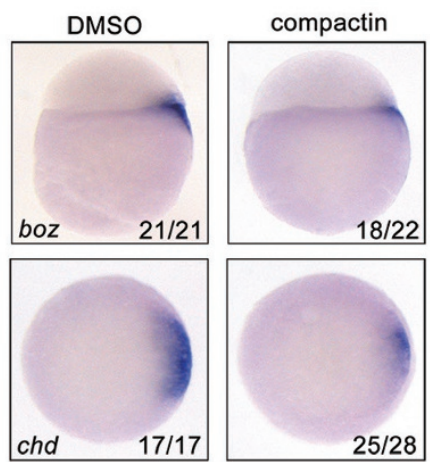

$\mathbf{F}$
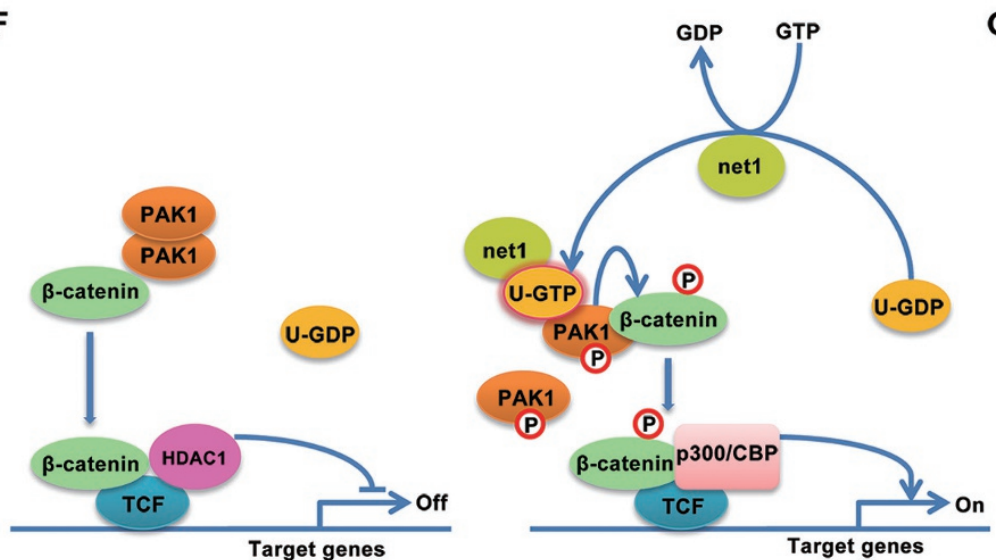

H

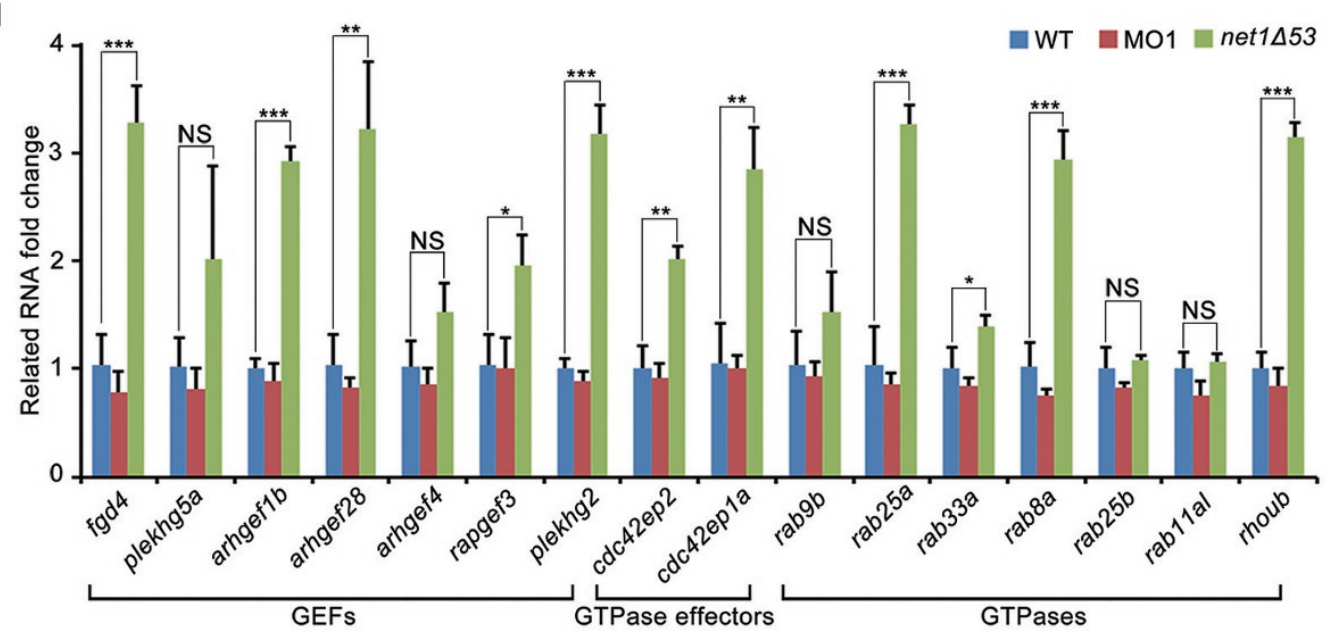

E
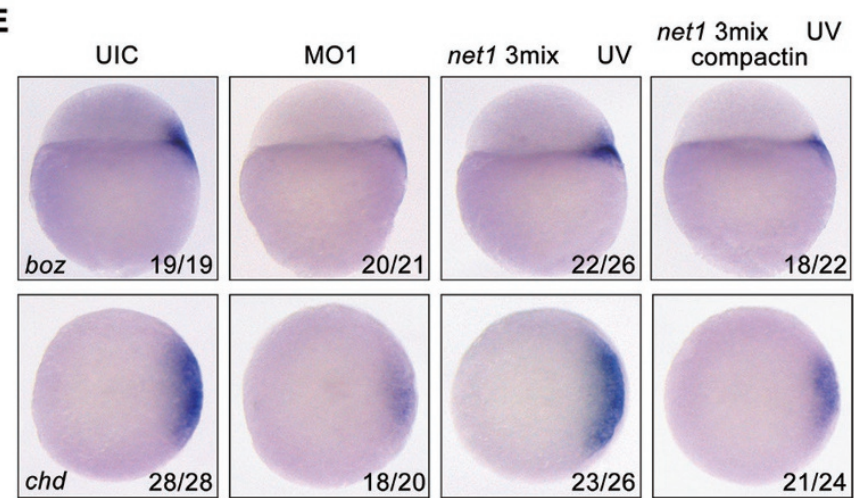

$\mathbf{G}_{\text {WT }}$ MO1 net1 153

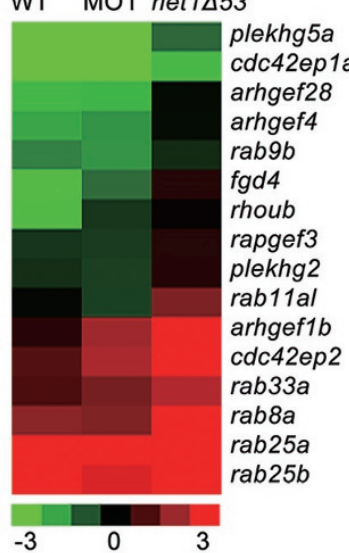

Myc-PAK $1=+;+$

Flag-PAK1 +-+++

HA-Net1 - - \begin{tabular}{cccc}
- & + & + \\
\hline & & + & + \\
\hline & 0.37 & 1.19
\end{tabular}
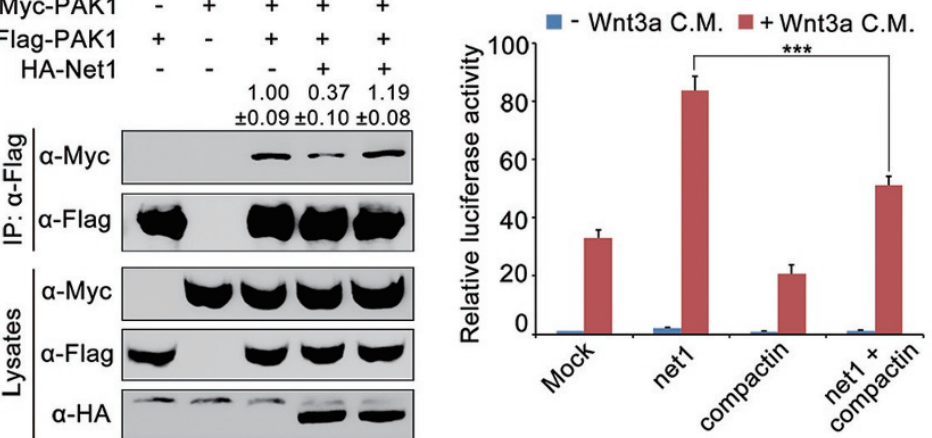

WT MO1 net1 $1 \Delta 53$

www.cell-research.com | Cell Research | SPRINGER NATURE 
Previous studies have shown that the expression of net 1 is rapidly induced by TGF- $\beta /$ Smad signaling in several human and mouse cell lines $[58,78]$. Our previous work also indicated that zebrafish net 1 is a direct downstream target of Nodal/Smad2 signaling at the onset of gastrulation [32]. In current study, we found that Nodal signal activates the expression of net 1 in the pre-dorsal organizer from sphere stage to shield stage. In the early zebrafish embryo, a dorsal to ventral gradient of Nodal activity is established [79]. As net1 exhibits highest expression level in the dorsal side but much weaker expression level in the lateral margin, we hypothesize that net 1 is a high-threshold-activated gene in response to Nodal signaling. Interestingly, Nodal-deficient embryos exhibit a normal phosphorylation level of $\beta$-catenin S675. It is likely that some Nodal-suppressed genes might be upregulated to maintain $\beta$-catenin phosphorylation in Nodal-deficient embryos. In agreement with these findings, the dorsal axis still forms in embryos lacking Nodal signaling [79-82].

Overexpression of netl can rescue the dorsal fate defects in net 1 morphants and result in markedly enlarged expression domains of dorsal marker genes in wild-type embryos. Furthermore, inactivation of zebrafish net 1 by an alternative technique, siRNA-based gene silencing, induces similar dorsal fate defects to those observed in net 1 morphants. In addition, knockdown of endogenous net 1 expression by shRNAs in mammalian cells causes a significant decrease in $\mathrm{Wnt} / \beta$-catenin signaling activity and target gene expression. Because these different knockdown strategies lead to similar effects in zebrafish embryos and mammalian cells, it is likely that any potential off-target effects in net 1 knockdown experiments are minimal.

We also generated net 1 null mutants using the Cas9/ gRNA system. Surprisingly, net 1 mutants display no obvious developmental defects; and are also, importantly, insensitive to the injection of net 1 MOs or siRNAs. Previous biochemical and genetic studies as well as clinical reports indicate that compensatory changes may allow organisms to adapt to genetic mutations [77, 83, 84]. Such properties have also been described in zebrafish genetic studies. Embryos injected with egfl7 morpholinos exhibit severe vascular defects, while egfl7 mutants do not show any obvious phenotypes, as a set of genes are upregulated to compensate for the induced genetic alteration [76]. Interestingly, we also observed a clear upregulation of a subset of genes encoding GEFs, GTPases and GTPase effectors in net 1 mutants. These net 1 mutants are still sensitive to compactin treatment or PAK1 activity inhibition, strongly suggesting that upregulation of these GTPase-mediated signal transduction-related genes compensates for the loss of Net1 function during embryonic development. Thus, our findings provide another example of compensatory gene regulation in response to an altered genetic environment.

Mammalian Net1 exists in two isoforms, Net1 and Net1A, which are produced by alternative splicing and differ only in their N-terminus. Both isoforms are mainly localized in the nucleus in quiescent cells, thereby preventing them from activating RhoA $[35,36]$, and their presence in the cytoplasm is tightly controlled. TGF- $\beta /$ Smad signaling has been reported to induce not only Net1 expression, but also its cytoplasmic localization, which is required for TGF- $\beta$-induced EMT of human retinal pigment epithelial cells [58]. Furthermore, active Rac1 stimulates Net1A re-localization to the cytoplasm by promoting acetylation on the N-terminal sites [85]. Although our study clearly demonstrates that both cytoplasmic and nuclear Netl can efficiently enhance canonical Wnt signaling and dorsal axis formation, it remains to be seen if zebrafish Net1 has alternatively spliced isoforms and whether these isoforms are localized to the cytoplasm during embryonic development.

Our study suggests a role of Net1 in regulating the transcriptional activity of $\beta$-catenin. During vertebrate embryonic development, maternal $\beta$-catenin protein, the transcriptional effector of canonical Wnt signaling, is stabilized in dorsal blastomeres by asymmetrically localized Wnt ligands to initiate the dorsal-ventral axis $[1,2]$. In zebrafish, the maternal wnt $8 a$ mRNA is transported from the vegetal pole to the future dorsal margin by a microtubule-dependent mechanism, which then leads to $\beta$-catenin nuclear translocation [14]. Zebrafish maternal $\beta$-catenin accumulates in the nuclei of dorsal marginal blastomeres as early as the 128 -cell stage $(2.25 \mathrm{hpf})$ $[81,86]$, but activates the transcription of dorsal genes including boz and chd only after the MBT (about $3 \mathrm{hpf}$ ) to inhibit the action of ventralizing Bmp signals [2]. The mechanism by which the transcriptional activity of maternal $\beta$-catenin is inhibited before MBT remains to be elucidated. Recent evidence suggests that alterations in DNA methylation and histone modifications around the time of MBT are important for zygotic gene activation [47, 87-90]. Similarly, $\beta$-catenin recruits the arginine methyltransferase Prmt2 to target promoters, thereby establishing poised chromatin architecture and priming organizer gene expression [91]. Whether the transcriptional activity of maternal $\beta$-catenin is controlled by internal self-regulating protein modifications remains unknown.

Our findings suggest that Net1 is essential for $\beta$-catenin activation to pattern the zebrafish embryonic body plan. Interestingly, we found that the relative phosphorylation level of $\beta$-catenin S675 is very low before MBT, 
but becomes significantly increased during and after MBT. The expression of net 1 results in the phosphorylation at $\mathrm{S} 675$, but not protein stability of $\beta$-catenin. Importantly, overexpression of $\beta$-catenin S675D but not wildtype $\beta$-catenin could lead to a much earlier expression of the dorsal marker genes. These observations suggest that $\beta$-catenin S675 phosphorylation, which is regulated by Net1, is a key requirement for the transcriptional activity of maternal $\beta$-catenin to trigger target gene expression and establish dorsal axis in zebrafish embryos. Whether the $\mathrm{C}$-terminal phosphorylation of $\beta$-catenin at other sites plays additional roles in embryonic patterning remains to be determined.

Multiple serine residues within the $\mathrm{C}$ terminus of $\beta$-catenin can be phosphorylated by various protein kinases. In particular, the $\mathrm{S} 675$ residue is a common target of PAK1 and PKA [22, 23, 60]. Our biochemical and genetic studies in zebrafish embryos and mammalian cells demonstrate that Net1 acts upstream of PAK1, but not PKA, to control $\beta$-catenin phosphorylation and dorsal axis formation. This premise is further supported because Net1 requires its GEF activity to promote $\beta$-catenin phosphorylation, and the kinase activity of PAK1 is regulated by GTP-loaded GTPases [65]. Interestingly, our results suggest that Net1 dissociates PAK1 homodimers thereby initiating PAK1 kinase activity to phosphorylate $\beta$-catenin in a GEF-dependent manner. Net1 specifically interacts with both GTP- and GDP-bound RhoA and catalyzes GDP exchange on RhoA, but not Rac1 or Cdc42 $[34,68]$. Moreover, our results, as well as those from previous genetic studies, indicate that RhoA mediates cell movements during zebrafish gastrulation but not cell fate determination [41, 92, 93]. Conversely, intracellular GTP-Rac1 and GTP-cdc42, but not activated RhoA, are known to disrupt PAK1 dimerization, thereby controlling $\beta$-catenin S675 phosphorylation and Wnt signal transduction $[23,65]$. Because Net1 GEF activity is required for regulating PAK1 homodimer dissociation, $\mathrm{Wnt} / \beta$-catenin signal transduction and zebrafish dorsal axis formation, we propose that a previously unidentified GTPase exists and is activated by Net1 to regulate downstream Wnt/ $\beta$-catenin signaling and dorsal patterning. Compactin, a pan Rho family GTPase inhibitor, was then used to reduce endogenous GTPase activity in mammalian cells and zebrafish embryos. Compactin treatment dramatically reduced the association between Net1 and PAK1, inhibited the ability of Net1 to disrupt PAK1 dimerization, suppressed Net1-induced Wnt/ $\beta$-catenin signal activity, and erased the rescue effect of net 1 mRNA on dorsal axis formation in net 1 morphants. These data confirm the requirement of Rho family GTPase activity in Net1-regulated canonical Wnt signal transduction and dorsal fate specification. We have tried pull-down experiments and yeast two-hybrid screening using full-length zebrafish Net1 as bait to identify Net1-regulated GTPases, but did not find any useful clue. Interestingly, a recent study found that PAK1 phosphorylates Net1 on three serine residues and negatively regulates its GEF activity [94], suggesting a negative-feedback loop in Net1-regulated Wnt signal activation.

In summary, our results reveal a conserved regulatory cascade of Net1-GTPase-PAK1 that controls canonical Wnt signaling in zebrafish embryos and mammalian cells. Future studies may determine whether this regulatory cascade similarly functions in the dorsal axis formation of other vertebrate animals. In addition, because upregulated net 1 expression and aberrant $\mathrm{Wnt} / \beta$-catenin signaling are widely implicated in numerous cancers $[12$, 42-46], it will be important to investigate the functional interaction of Net1 and Wnt/ $\beta$-catenin signaling in cancer cell proliferation, motility and invasiveness.

\section{Materials and Methods}

\section{Zebrafish strains}

Embryos and adult fish were raised and maintained under standard laboratory conditions. Embryos were staged by morphology as previously described [95]. Homozygous p53(M214K) mutant embryos (abbreviated as $p 53^{-/-}$) carrying a loss-of-function $p 53$ point mutation were employed as controls to account for any non-specific cell apoptosis induced by morpholino injection. TOP$d G F P$ transgenic embryos were used to confirm the endogenous activity of $\mathrm{Wnt} / \beta$-catenin signaling during embryonic development. Our studies involving zebrafish embryo collection and analyses were in full compliance with the Regulations for the Care and Use of Laboratory Animals by the Ministry of Science and Technology of China, and with the Institute of Zoology's Guidelines for the Care and Use of Laboratory Animals. The experimental protocol was approved by the Animal Care and Use Committee at the Institute of Zoology, Chinese Academy of Sciences (Permission Number: IOZ-13048).

\section{Cell lines and transfection}

HEK293T, MCF-7, Hela and L cell lines (American Tissue Culture Collection, ATCC, USA) were cultured in DMEM medium supplemented with $10 \% \mathrm{FBS}$ in a $37{ }^{\circ} \mathrm{C}$ humidified incubator in a $5 \% \mathrm{CO}_{2}$ environment. The Wnt3a-expressing $\mathrm{L}$ cell line was grown under similar conditions, but supplemented with $0.4 \mathrm{mg}$ / $\mathrm{ml} \mathrm{G-418} \mathrm{to} \mathrm{select} \mathrm{for} \mathrm{Wnt3a-expressing} \mathrm{cells.} \mathrm{The} \mathrm{Wnt3a} \mathrm{condi-}$ tioned medium (Wnt3a CM) was generated following the protocol of ATCC. For Wnt stimulation experiments, Wnt3a CM was mixed with normal culture medium at the volume ratio 1:1. Cell transfection was performed using Lipofectamine 2000 (11668019, Invitrogen) following the manufacturer's instructions.

\section{RNA and morpholino microinjection}

Capped mRNAs for zebrafish net 1 , wnt $8 a, \Delta N$ - $\beta$-catenin, $\beta$-catenin S675D, $\beta$-S675A, dnpakl, capakl and rhoA N19 were syn- 
thesized in vitro from corresponding linearized plasmids using the mMessage mMachine kit (Ambion). The following morpholinos were synthesized by Gene Tools and re-suspended in nuclease free water: net1 MO1 (5'-CTTGCTCCGGCTGTACTCACCTCTT-3'), net1 MO2 (5'-AAAGAATGTACAAACCTTTCGGGCT-3'), control MO (mis-MO1) (5'-CTTCCTGCGCCTGTAGTCACGTCTT-3') and AS-Flag-photo-MO (5'-TCATCGTCGTpCTTGTAGTCCAT-3'). Microinjection was performed as previously described. For net 1 mRNA overexpression experiments, embryos were co-injected with pre-mixed Flag-net 1 mRNA and AS-Flagphoto-MO (200 pg Flag-net1 mRNA and 1 ng AS-Flag-photo-MO per embryo) at 1-cell stage, then treated with $365 \mathrm{~nm}$ UV $10 \mathrm{~min}$ by Lightbox (Gene Tools, LLC) at 64-cell stage, and collected at sphere stage. All of these processes were conducted under constant dark conditions.

\section{Whole-mount in situ hybridization}

Digoxigenin-UTP-labeled antisense RNA probes were transcribed using MEGAscript Kit (Ambion) according to the manufacturer's instructions. Whole-mount in situ hybridizations (WISH) were performed according to previously published methods [32].

\section{Embryonic treatment}

To block RhoA activity, embryos were injected with $1 \mathrm{nl}$ of 20 $\mu \mathrm{M}$ C. botulinum toxin C3 transferase (C3) (A8724, Sigma) at the 1-cell stage, then collected at sphere stage for WISH. To disrupt Ras family GTPase activity, embryos were treated with $10 \mu \mathrm{M}$ compactin (M2537, Sigma) at the 16-cell stage, then harvested at the sphere stage for subsequent experiments. To inhibit PAK1 activity, embryos were treated with $10 \mu \mathrm{M}$ IPA-3 (I2285, Sigma) at the 1-cell stage, and then subjected to in situ hybridization at sphere stage. To repress PKA activity, embryos were treated with $10 \mu \mathrm{M} H 89$ dihydrochloride hydrate (B1427, Sigma) at the 16-cell stage, and collected at sphere stage for WISH.

\section{Dual-luciferase reporter assays}

For the luciferase reporter assays, either HEK293T or MCF7 cells were transfected with super-TOPFlash and the indicated plasmids. The Renilla luciferase reporter was used as an internal control. Cells were stimulated with or without Wnt3a CM for 12 $\mathrm{h}$ before harvesting for luciferase activity assays. Each luciferase reporter assay was repeated at least three times, and the average activity was calculated.

\section{Immunoprecipitation and western blot analysis}

For immunoprecipitation assays, HEK293T cells were transfected with the indicated plasmids. Cells were harvested $48 \mathrm{~h}$ after transfection and lysed with TNE lysis buffer (10 mM Tris- $\mathrm{HCl}, \mathrm{pH}$ $7.5,150 \mathrm{mM} \mathrm{NaCl}, 2 \mathrm{mM}$ EDTA and $0.5 \%$ Nonidet P-40) containing a protease inhibitor cocktail. Lysates were subjected to immunoprecipitation assay as previously described [32].

For immunoblotting, we used affinity-purified anti-Flag (F2555, Sigma), anti-HA (CW0092A, CW), anti-Myc (M047-3, MBL), anti- $\beta$-catenin (M24002, Abmart), anti-Phospho- $\beta$-catenin (Ser675) (4176, Cell Signaling Technology), anti-PAK1 (2602, Cell Signaling Technology), anti-Phospho-PAK1 (Thr423) (2601, Cell Signaling Technology), anti-Net1(AI13372, Abgent), anti-Phospho-Histone H3 (3377, Cell Signaling Technology), anti-GFP (A11120, Thermo Fisher) and anti-GST (M20007, Abmart) antibodies.

\section{Immunofluorescence analysis}

Cells on coverslips were fixed in $4 \%$ paraformaldehyde/PBS for $10 \mathrm{~min}$ at room temperature then permeabilized with $0.2 \%$ Triton $\mathrm{X}-100 / \mathrm{PBS}$ for $10 \mathrm{~min}$. After $30 \mathrm{~min}$ blocking in 1.5\% FBS/PBS, cells were incubated with the indicated antibodies for $1 \mathrm{~h}$ at room temperature or overnight at $4{ }^{\circ} \mathrm{C}$. 4',6-Diamidine-2'-phenylindole dihydrochloride (DAPI, 10236276001, Sigma) was used to counterstain nuclei. All immunofluoresence images within each experiment were captured using Nikon A1R+ confocal microscope under the same settings.

\section{Statistical analysis}

Student's $t$-test was performed to analyze all data sets (Microsoft Excel software). At a minimum, experiments were performed in triplicate. Results were considered statistically significant at $P<$ 0.05 .

\section{Acknowledgments}

This work was supported by grants from the National key research and development program (2016YFA0100503) and the National Natural Science Foundation of China (31322035, 31271532, 31571501 and 91519329).

\section{Author Contributions}

SW and QW conceived and designed the experiments and wrote the manuscript. SW performed most of the experiments and analysis. MD and ZL performed experiments presented in Figure $2 \mathrm{~F}-2 \mathrm{H}$. YM constructed net 1 shRNA vectors. HS and YC verified the functions of RhoA in zebrafish embryo development. QW supervised the entire project. All authors reviewed and commented on the manuscript.

\section{Competing Financial Interests}

The authors declare no conflict of interest.

\section{References}

1 De Robertis EM, Kuroda H. Dorsal-ventral patterning and neural induction in Xenopus embryos. Annu Rev Cell Dev Biol 2004; 20:285-308.

2 Schier AF, Talbot WS. Molecular genetics of axis formation in zebrafish. Annu Rev Genet 2005; 39:561-613.

3 Bellipanni G, Varga M, Maegawa S, et al. Essential and opposing roles of zebrafish $\beta$-catenins in the formation of dorsal axial structures and neurectoderm. Development 2006; 133:1299-1309.

4 Tao Q, Yokota C, Puck H, et al. Maternal wnt11 activates the canonical wnt signaling pathway required for axis formation in Xenopus embryos. Cell 2005; 120:857-871.

5 Heasman J, Crawford A, Goldstone K, et al. Overexpression of cadherins and underexpression of $\beta$-catenin inhibit dorsal mesoderm induction in early Xenopus embryos. Cell 1994; 79:791-803.

6 Kelly C, Chin AJ, Leatherman JL, Kozlowski DJ, Weinberg ES. Maternally controlled $\beta$-catenin-mediated signaling is required for organizer formation in the zebrafish. Development 2000; 127:3899-3911. 
7 Weaver C, Kimelman D. Move it or lose it: axis specification in Xenopus. Development 2004; 131:3491-3499.

8 Xiong B, Rui Y, Zhang M, et al. Tobl controls dorsal development of zebrafish embryos by antagonizing maternal $\beta$-catenin transcriptional activity. Dev Cell 2006; 11:225-238.

9 Rubinfeld B, Albert I, Porfiri E, Fiol C, Munemitsu S, Polakis P. Binding of GSK $3 \beta$ to the APC- $\beta$-catenin complex and regulation of complex assembly. Science 1996; 272:1023-1026.

10 Aberle H, Bauer A, Stappert J, Kispert A, Kemler R. $\beta$-catenin is a target for the ubiquitin-proteasome pathway. EMBO J 1997; 16:3797-3804.

11 Liu C, Li Y, Semenov M, et al. Control of $\beta$-catenin phosphorylation/degradation by a dual-kinase mechanism. Cell 2002; 108:837-847.

12 Moon RT, Kohn AD, De Ferrari GV, Kaykas A. WNT and $\beta$-catenin signaling: diseases and therapies. Nat Rev Genet 2004; 5:691-701.

13 Mosimann C, Hausmann G, Basler K. B-catenin hits chromatin: regulation of Wnt target gene activation. Nat Rev Mol Cell Biol 2009; 10:276-286.

14 Lu FI, Thisse C, Thisse B. Identification and mechanism of regulation of the zebrafish dorsal determinant. Proc Natl Acad Sci USA 2011; 108:15876-15880.

15 Yang J, Tan C, Darken RS, Wilson PA, Klein PS. $\beta$-catenin/ Tcf-regulated transcription prior to the midblastula transition. Development 2002; 129:5743-5752.

16 Dosch R, Wagner DS, Mintzer KA, Runke G, Wiemelt AP, Mullins MC. Maternal control of vertebrate development before the midblastula transition: mutants from the zebrafish I. Dev Cell 2004; 6:771-780.

17 Fekany K, Yamanaka Y, Leung T, et al. The zebrafish bozozok locus encodes Dharma, a homeodomain protein essential for induction of gastrula organizer and dorsoanterior embryonic structures. Development 1999; 126:1427-1438.

18 Piccolo S, Sasai Y, Lu B, De Robertis EM. Dorsoventral patterning in Xenopus: inhibition of ventral signals by direct binding of chordin to BMP-4. Cell 1996; 86:589-598.

19 Schulte-Merker S, Lee KJ, McMahon AP, Hammerschmidt M. The zebrafish organizer requires chordino. Nature 1997; 387:862-863.

20 Park MH, Kim DJ, You ST, et al. Phosphorylation of $\beta$-catenin at serine 663 regulates its transcriptional activity. Biochem Biophys Res Commun 2012; 419:543-549.

21 Fang D, Hawke D, Zheng Y, et al. Phosphorylation of $\beta$-catenin by AKT promotes $\beta$-catenin transcriptional activity. $J$ Biol Chem 2007; 282:11221-11229.

22 Taurin S, Sandbo N, Qin Y, Browning D, Dulin NO. Phosphorylation of $\beta$-catenin by cyclic AMP-dependent protein kinase. J Biol Chem 2006; 281:9971-9976.

23 Zhu G, Wang Y, Huang B, et al. A Rac1/PAK1 cascade controls $\beta$-catenin activation in colon cancer cells. Oncogene 2012; 31:1001-1012.

24 Piedra J, Martinez D, Castano J, Miravet S, Dunach M, de Herreros AG. Regulation of $\beta$-catenin structure and activity by tyrosine phosphorylation. J Biol Chem 2001; 276:2043620443.

25 Hall A, Nobes CD. Rho GTPases: molecular switches that control the organization and dynamics of the actin cytoskeleton. Philos Trans R Soc Lond B Biol Sci 2000; 355:965-970.
26 Schmidt A, Hall MN. Signaling to the actin cytoskeleton. Annu Rev Cell Dev Biol 1998; 14:305-338.

$27 \mathrm{Wu}$ X, Tu X, Joeng KS, Hilton MJ, Williams DA, Long F. Rac1 activation controls nuclear localization of $\beta$-catenin during canonical Wnt signaling. Cell 2008; 133:340-353.

28 Buongiorno P, Pethe VV, Charames GS, Esufali S, Bapat B. Rac1 GTPase and the Rac1 exchange factor Tiam1 associate with Wnt-responsive promoters to enhance $\beta$-catenin/TCF-dependent transcription in colorectal cancer cells. Mol Cancer 2008; 7:73.

29 Upadhyay G, Goessling W, North TE, Xavier R, Zon LI, Yajnik V. Molecular association between $\beta$-catenin degradation complex and Rac guanine exchange factor DOCK4 is essential for Wnt/ $\beta$-catenin signaling. Oncogene 2008; 27:58455855.

30 Chahdi A, Raufman JP. The Cdc42/Rac nucleotide exchange factor protein $\beta 1 \mathrm{Pix}$ (Pak-interacting exchange factor) modulates $\beta$-catenin transcriptional activity in colon cancer cells: evidence for direct interaction of $\beta 1$ PIX with $\beta$-catenin. $J$ Biol Chem 2013; 288:34019-34029.

31 Greer ER, Chao AT, Bejsovec A. Pebble/ECT2 RhoGEF negatively regulates the Wingless/Wnt signaling pathway. Development 2013; 140:4937-4946.

32 Liu Z, Lin X, Cai Z, et al. Global identification of SMAD2 target genes reveals a role for multiple co-regulatory factors in zebrafish early gastrulas. J Biol Chem 2011; 286:2852028532.

33 Chan AM, Takai S, Yamada K, Miki T. Isolation of a novel oncogene, NET1, from neuroepithelioma cells by expression cDNA cloning. Oncogene 1996; 12:1259-1266.

34 Alberts AS, Treisman R. Activation of RhoA and SAPK/JNK signalling pathways by the RhoA-specific exchange factor mNET1. EMBO J 1998; 17:4075-4085.

35 Schmidt A, Hall A. The Rho exchange factor Net1 is regulated by nuclear sequestration. $J$ Biol Chem 2002; 277:1458114588.

36 Qin H, Carr HS, Wu X, Muallem D, Tran NH, Frost JA. Characterization of the biochemical and transforming properties of the neuroepithelial transforming protein 1. J Biol Chem 2005; 280:7603-7613.

37 Srougi MC, Burridge K. The nuclear guanine nucleotide exchange factors Ect2 and Net1 regulate RhoB-mediated cell death after DNA damage. PLoS One 2011; 6:e17108.

38 Miyakoshi A, Ueno N, Kinoshita N. Rho guanine nucleotide exchange factor xNET1 implicated in gastrulation movements during Xenopus development. Differentiation 2004; 72:48-55.

39 Nakaya Y, Sukowati EW, Wu Y, Sheng G. RhoA and microtubule dynamics control cell-basement membrane interaction in EMT during gastrulation. Nat Cell Biol 2008; 10:765-775.

40 Zuo Y, Berdeaux R, Frost JA. The RhoGEF Net1 is required for normal mammary gland development. Mol Endocrinol 2014; 28:1948-1960.

41 Zhu S, Liu L, Korzh V, Gong Z, Low BC. RhoA acts downstream of Wnt5 and Wnt11 to regulate convergence and extension movements by involving effectors Rho kinase and Diaphanous: use of zebrafish as an in vivo model for GTPase signaling. Cell Signal 2006; 18:359-372.

42 Leyden J, Murray D, Moss A, et al. Net1 and Myeov: computationally identified mediators of gastric cancer. $\mathrm{Br} J$ Cancer 
2006; 94:1204-1212.

43 Shen SQ, Li K, Zhu N, Nakao A. Expression and clinical significance of NET-1 and PCNA in hepatocellular carcinoma. Med Oncol 2008; 25:341-345.

$44 \mathrm{Tu}$ Y, Lu J, Fu J, et al. Over-expression of neuroepithelial-transforming protein 1 confers poor prognosis of patients with gliomas. Jpn J Clin Oncol 2010; 40:388-394.

45 Gilcrease MZ, Kilpatrick SK, Woodward WA, et al. Coexpression of $\alpha 6 \beta 4$ integrin and guanine nucleotide exchange factor Net1 identifies node-positive breast cancer patients at high risk for distant metastasis. Cancer Epidemiol Biomarkers Prev 2009; 18:80-86.

46 Murray D, Horgan G, Macmathuna P, Doran P. NET1-mediated RhoA activation facilitates lysophosphatidic acid-induced cell migration and invasion in gastric cancer. $\mathrm{Br} J$ Cancer 2008; 99:1322-1329.

47 Wu D, Chen L, Sun Q, Wu X, Jia S, Meng A. Uracil-DNA glycosylase is involved in DNA demethylation and required for embryonic development in the zebrafish embryo. $J$ Biol Chem 2014; 289:15463-15473.

48 Dodd A, Chambers SP, Love DR. Short interfering RNA-mediated gene targeting in the zebrafish. FEBS Lett 2004; 561:89-93.

49 Liu WY, Wang Y, Sun YH, Wang YP, Chen SP, Zhu ZY. Efficient RNA interference in zebrafish embryos using siRNA synthesized with SP6 RNA polymerase. Dev Growth Differ 2005; 47:323-331.

50 Ma Y, Liu X, Liu Z, et al. The chromatin remodeling protein Bptf promotes posterior neuroectodermal fate by enhancing Smad2-activated wnt8a expression. J Neurosci 2015; 35:8493-8506.

51 Leung T, Soll I, Arnold SJ, Kemler R, Driever W. Direct binding of Lef1 to sites in the boz promoter may mediate pre-midblastula-transition activation of boz expression. Dev Dyn 2003; 228:424-432.

52 Li Z, Nie F, Wang S, Li L. Histone H4 Lys 20 monomethylation by histone methylase SET8 mediates Wnt target gene activation. Proc Natl Acad Sci USA 2011; 108:3116-3123.

53 Ro H, Dawid IB. Modulation of Tcf3 repressor complex composition regulates cdx 4 expression in zebrafish. EMBO J 2011; 30:2894-2907.

54 Szeto DP, Kimelman D. Combinatorial gene regulation by Bmp and Wnt in zebrafish posterior mesoderm formation. Development 2004; 131:3751-3760.

55 Dorsky RI, Sheldahl LC, Moon RT. A transgenic Lef1/ $\beta$-catenin-dependent reporter is expressed in spatially restricted domains throughout zebrafish development. Dev Biol 2002; 241:229-237.

56 Lekven AC, Thorpe CJ, Waxman JS, Moon RT. Zebrafish wnt 8 encodes two wnt 8 proteins on a bicistronic transcript and is required for mesoderm and neurectoderm patterning. Dev Cell 2001; 1:103-114.

57 Garcia-Mata R, Dubash AD, Sharek L, Carr HS, Frost JA, Burridge K. The nuclear RhoA exchange factor Net1 interacts with proteins of the Dlg family, affects their localization, and influences their tumor suppressor activity. Mol Cell Biol 2007; 27:8683-8697.

58 Lee J, Moon HJ, Lee JM, Joo CK. Smad3 regulates Rho signaling via NET1 in the transforming growth factor- $\beta$-induced epithelial-mesenchymal transition of human retinal pigment epithelial cells. J Biol Chem 2010; 285:26618-26627.

59 Meijer L, Skaltsounis AL, Magiatis P, et al. GSK-3-selective inhibitors derived from Tyrian purple indirubins. Chem Biol 2003; 10:1255-1266.

60 Spirli C, Locatelli L, Morell CM, et al. Protein kinase A-dependent $\mathrm{pSer}(675)-\beta$-catenin, a novel signaling defect in a mouse model of congenital hepatic fibrosis. Hepatology 2013; 58:1713-1723.

61 Lee SH, Jung YS, Chung JY, et al. Novel tumor suppressive function of Smad4 in serum starvation-induced cell death through PAK1-PUMA pathway. Cell Death Dis 2011; 2:e235.

62 Hogarth DK, Sandbo N, Taurin S, Kolenko V, Miano JM, Dulin NO. Dual role of PKA in phenotypic modulation of vascular smooth muscle cells by extracellular ATP. Am J Physiol Cell Physiol 2004; 287:C449-456.

63 Chijiwa T, Mishima A, Hagiwara M, et al. Inhibition of forskolin-induced neurite outgrowth and protein phosphorylation by a newly synthesized selective inhibitor of cyclic AMP-dependent protein kinase, $\mathrm{N}$-[2-(p-bromocinnamylamino)ethyl]-5-isoquinolinesulfonamide (H-89), of PC12D pheochromocytoma cells. J Biol Chem 1990; 265:5267-5272.

64 Song BH, Choi SC, Han JK. Local activation of protein kinase A inhibits morphogenetic movements during Xenopus gastrulation. Dev Dyn 2003; 227:91-103.

65 Parrini MC, Lei M, Harrison SC, Mayer BJ. Pak1 kinase homodimers are autoinhibited in trans and dissociated upon activation by Cdc42 and Rac1. Mol Cell 2002; 9:73-83.

66 Billin AN, Thirlwell H, Ayer DE. $\beta$-catenin-histone deacetylase interactions regulate the transition of LEF 1 from a transcriptional repressor to an activator. Mol Cell Biol 2000; 20:6882-6890.

67 Lei M, Lu W, Meng W, et al. Structure of PAK1 in an autoinhibited conformation reveals a multistage activation switch. Cell 2000; 102:387-397.

68 Alberts AS, Bouquin N, Johnston LH, Treisman R. Analysis of RhoA-binding proteins reveals an interaction domain conserved in heterotrimeric $G$ protein $\beta$ subunits and the yeast response regulator protein Skn7. J Biol Chem 1998; 273:86168622.

69 Bagrodia S, Taylor SJ, Creasy CL, Chernoff J, Cerione RA. Identification of a mouse p21Cdc42/Rac activated kinase. $J$ Biol Chem 1995; 270:22731-22737.

70 Manser E, Leung T, Salihuddin H, Zhao ZS, Lim L. A brain serine/threonine protein kinase activated by Cdc42 and Rac1. Nature 1994; 367:40-46.

71 Reid T, Furuyashiki T, Ishizaki T, et al. Rhotekin, a new putative target for Rho bearing homology to a serine/threonine kinase, PKN, and rhophilin in the rho-binding domain. $J$ Biol Chem 1996; 271:13556-13560.

72 Rossol-Allison J, Stemmle LN, Swenson-Fields KI, et al. Rho GTPase activity modulates Wnt $3 \mathrm{a} / \beta$-catenin signaling. Cell Signal 2009; 21:1559-1568.

73 Rodrigues P, Macaya I, Bazzocco S, et al. RHOA inactivation enhances Wnt signalling and promotes colorectal cancer. Nat Commun 2014; 5:5458.

74 Seabra MC. Membrane association and targeting of prenylated Ras-like GTPases. Cell Signal 1998; 10:167-172.

75 Chang N, Sun C, Gao L, et al. Genome editing with 
RNA-guided Cas 9 nuclease in zebrafish embryos. Cell Res 2013; 23:465-472.

76 Rossi A, Kontarakis Z, Gerri C, et al. Genetic compensation induced by deleterious mutations but not gene knockdowns. Nature 2015; 524:230-233.

77 Carelle-Calmels N, Saugier-Veber P, Girard-Lemaire F, et al. Genetic compensation in a human genomic disorder. $N$ Engl $J$ Med 2009; 360:1211-1216.

78 Shen X, Li J, Hu PP, Waddell D, Zhang J, Wang XF. The activity of guanine exchange factor NET1 is essential for transforming growth factor- $\beta$-mediated stress fiber formation. $J$ Biol Chem 2001; 276:15362-15368.

79 Feldman B, Gates MA, Egan ES, et al. Zebrafish organizer development and germ-layer formation require nodal-related signals. Nature 1998; 395:181-185.

80 Gritsman K, Zhang J, Cheng S, Heckscher E, Talbot WS, Schier AF. The EGF-CFC protein one-eyed pinhead is essential for nodal signaling. Cell 1999; 97:121-132.

81 Dougan ST, Warga RM, Kane DA, Schier AF, Talbot WS. The role of the zebrafish nodal-related genes squint and cyclops in patterning of mesendoderm. Development 2003; 130:18371851.

82 Hagos EG, Fan X, Dougan ST. The role of maternal Activin-like signals in zebrafish embryos. Dev Biol 2007; 309:245258.

83 Greenspan RJ. The flexible genome. Nat Rev Genet 2001; 2:383-387.

84 Marder E, Goaillard JM. Variability, compensation and homeostasis in neuron and network function. Nat Rev Neurosci 2006; 7:563-574.

85 Song EH, Oh W, Ulu A, Carr HS, Zuo Y, Frost JA. Acetylation of the RhoA GEF Net1A controls its subcellular localization and activity. J Cell Sci 2015; 128:913-922.
86 Schneider S, Steinbeisser H, Warga RM, Hausen P. $\beta$-catenin translocation into nuclei demarcates the dorsalizing centers in frog and fish embryos. Mech Dev 1996; 57:191-198.

87 Andersen IS, Ostrup O, Lindeman LC, et al. Epigenetic complexity during the zebrafish mid-blastula transition. Biochem Biophys Res Commun 2012; 417:1139-1144.

88 Lindeman LC, Andersen IS, Reiner AH, et al. Prepatterning of developmental gene expression by modified histones before zygotic genome activation. Dev Cell 2011; 21:993-1004.

89 Vastenhouw NL, Zhang Y, Woods IG, et al. Chromatin signature of embryonic pluripotency is established during genome activation. Nature 2010; 464:922-926.

90 Aday AW, Zhu LJ, Lakshmanan A, Wang J, Lawson ND. Identification of cis regulatory features in the embryonic zebrafish genome through large-scale profiling of $\mathrm{H} 3 \mathrm{~K} 4 \mathrm{me} 1$ and H3K4me3 binding sites. Dev Biol 2011; 357:450-462.

91 Blythe SA, Cha SW, Tadjuidje E, Heasman J, Klein PS. $\beta$-Catenin primes organizer gene expression by recruiting a histone $\mathrm{H} 3$ arginine 8 methyltransferase, Prmt2. Dev Cell 2010; 19:220-231.

92 Lai SL, Chang CN, Wang PJ, Lee SJ. Rho mediates cytokinesis and epiboly via ROCK in zebrafish. Mol Reprod Dev 2005; 71:186-196.

93 Weiser DC, Row RH, Kimelman D. Rho-regulated myosin phosphatase establishes the level of protrusive activity required for cell movements during zebrafish gastrulation. Development 2009 ; 136:2375-2384.

94 Alberts AS, Qin H, Carr HS, Frost JA. PAK1 negatively regulates the activity of the Rho exchange factor NET1. J Biol Chem 2005; 280:12152-12161.

95 Kimmel CB, Ballard WW, Kimmel SR, Ullmann B, Schilling TF. Stages of embryonic development of the zebrafish. Dev Dyn 1995; 203:253-310.

(Supplementary information is linked to the online version of the paper on the Cell Research website.) 